San Jose State University

SJSU ScholarWorks

Doctoral Projects

Master's Theses and Graduate Research

Spring 5-1-2015

\title{
Chinese Community Physicians' Perception Toward Collaboration with Advanced Practice Nurses
}

Ivy Tan

Northern California Consortium, Doctor of Nursing Practice Program, California State University, Fresno and San José State University

Follow this and additional works at: https://scholarworks.sjsu.edu/etd_doctoral

Part of the Family Practice Nursing Commons

\section{Recommended Citation}

Tan, Ivy, "Chinese Community Physicians' Perception Toward Collaboration with Advanced Practice Nurses" (2015). Doctoral Projects. 10.

DOI: https://doi.org/10.31979/etd.usjq-3xcm

https://scholarworks.sjsu.edu/etd_doctoral/10

This Doctoral Project is brought to you for free and open access by the Master's Theses and Graduate Research at SJSU ScholarWorks. It has been accepted for inclusion in Doctoral Projects by an authorized administrator of SJSU ScholarWorks. For more information, please contact scholarworks@sjsu.edu. 


\begin{abstract}
Effective interprofessional collaboration has been shown to be beneficial for both patients and providers. Yet collaboration between physicians and advanced practiced nurses (APNs) has been problematic. Little was known about such collaboration in an ethnic group like the Chinese community. This quantitative descriptive capstone project was to assess the perception among physicians, who are the members of the Chinese Community Healthcare Association in the San Francisco area, toward collaboration with APNs. The study also attempted to gain insight of collaborative attitudes of physicians in the Chinese community in order to fill the gap in the literature in this area. Data collection involved mail and online survey methods, using a modified Jefferson Scale of Attitudes toward physician-nurse collaboration and six researchergenerated questions. 44 out of 217 physicians participated in the study. Results indicated the physicians in the Chinese community carried the same attitude toward APNs as their colleagues from other ethnicities, and physician dominant authority was deeply rooted in this physician group. Gender or age seems to have no effect on physician's attitude, and primary care physicians tend to have a more positive attitude than the specialists. Personal, interpersonal and cultural influences seem to be relatively weak factors, yet the professional and organizational influences had more substantial effects on collaboration attitudes. Future studies are needed to better comprehend cultural or ethnicity's impact on providers' attitude toward collaboration.
\end{abstract}




\title{
CHINESE COMMUNITY PHYSICIANS' PERCEPTION TOWARD COLLABORATION WITH ADVANCED PRACTICE NURSES
}

\author{
By \\ Ivy Tan, DNP(c), RN, FNP-C
}

\begin{abstract}
A Project
Submitted to the Graduate Faculty as partial fulfillment of the requirements for the Degree of Doctor of Nursing Practice in the School of Nursing California State University, Fresno
\end{abstract}

April 2015 
APPROVED

For the Department of Nursing

We, the undersigned, certify that the thesis of the following student meets the required standards of scholarship, format, and style of the university and the student's graduate degree program for the awarding of the doctoral degree.

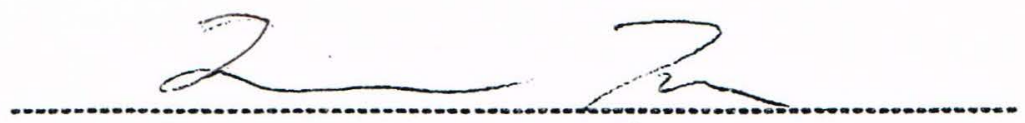

DNP project author
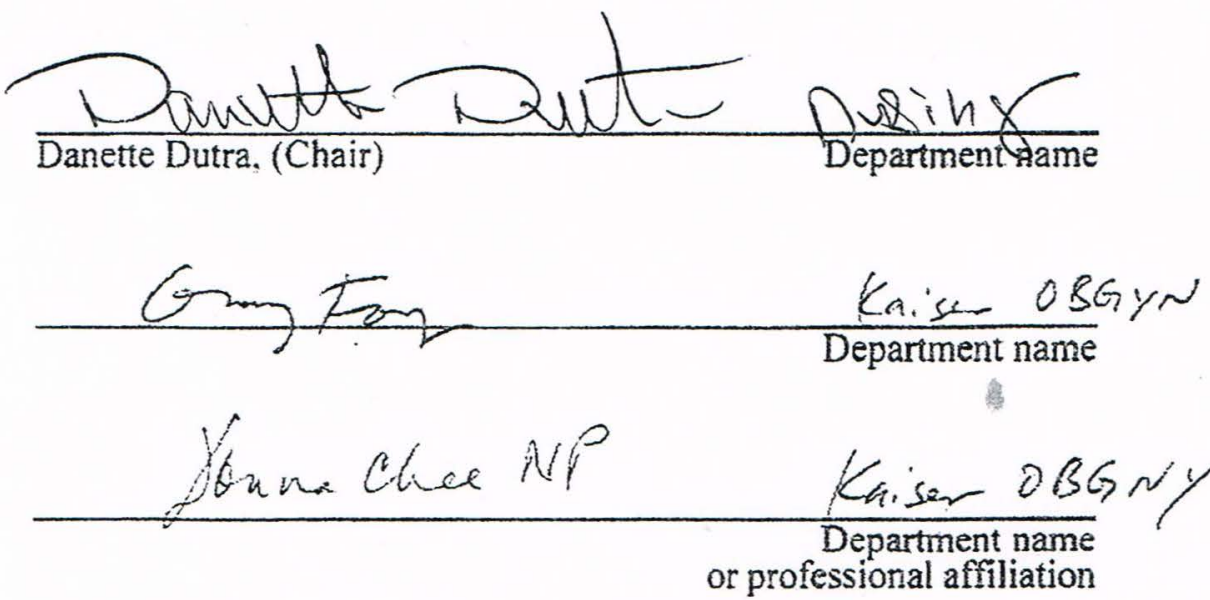


\section{AUTHORIZATION FOR REPRODUCTION \\ OF DOCTORAL CAPISTONE PROJECT}

I grant permission for the reproduction of this project in part or in its entirety without further authorization from me, on the condition that the person and agency requesting reproduction absorbs the cost and provides proper acknowledgement of authorship.

Permission to reproduce this project in part or in its entirety must be obtained from me.

Signature of project author

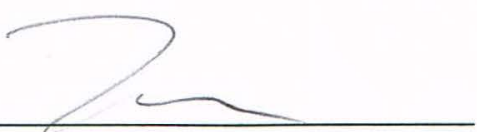




\section{Acknowledgements}

I wish to acknowledge the love and support given to me by my dearest husband George Tan, my lovely daughter, Chloe Tan, and my hard-working mother, Qi Ming Tan. Because of your unconditional love, I am able to remain on the course toward the completion of this doctoral capstone project.

To my project chair, Dr. Danette Dutra, the committee members, Dr. Ginny Fong and Ms. Donna Chee, who inspired my creativity, I am especially grateful. To Dr. Gregory Graham who provided expert statistical advice and Ms. Polly Chen and Dr. Clifford Chew who assisted me with contact information of the participating physicians, I offer special appreciation. And finally, to all the physicians who took the time out of their busy schedules to complete the survey, I extend a heartfelt thank you. 


\section{TABLE OF CONTENTS}

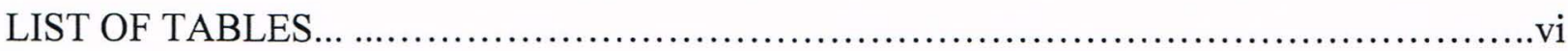

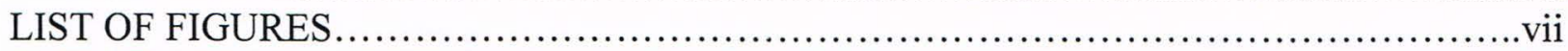

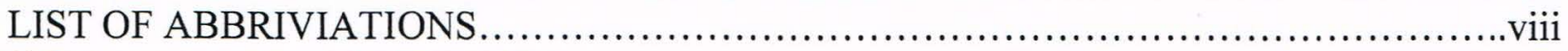

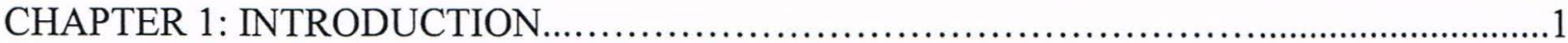

Problem Statement and Significance....................................................

Theoretical Framework.................................................................

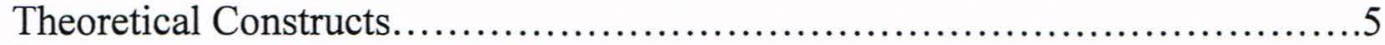

Collaborative Conceptual Models.............................................

CHAPTER 2: REVIEW OF LITERATURE................................................

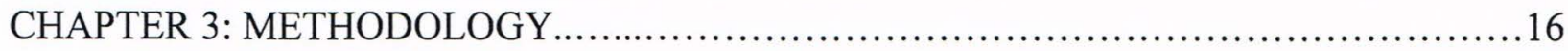

Setting/Organizational Structures..................................................

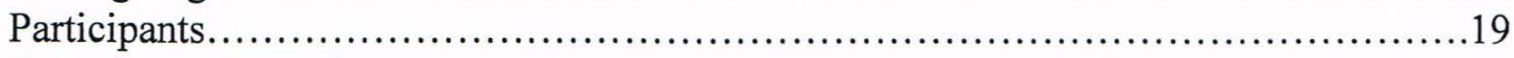

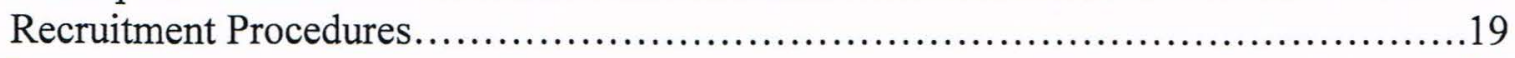

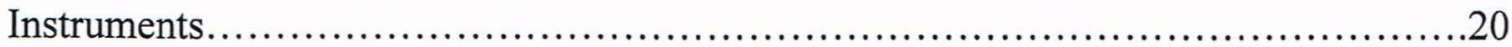

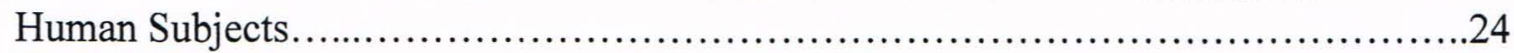

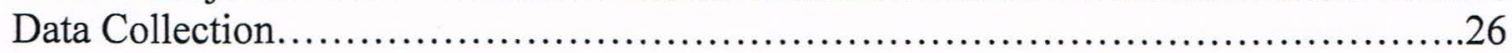

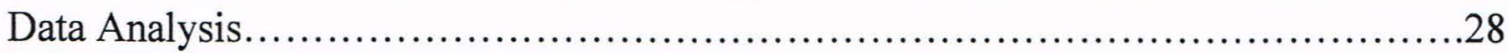

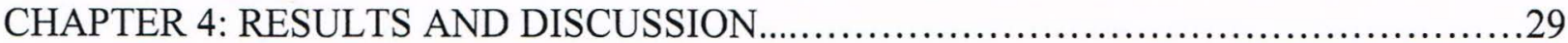

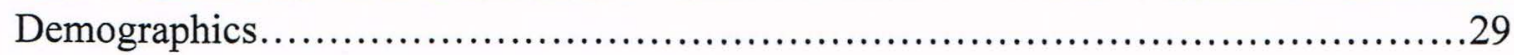

Researcher-generated Questions Analysis........................................ 31

Jefferson Scale of Attitudes Analysis.............................................. 33

Results of Jefferson scale survey.............................................33

Jefferson scale scores........................................................

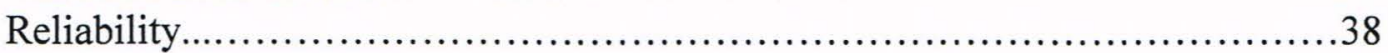

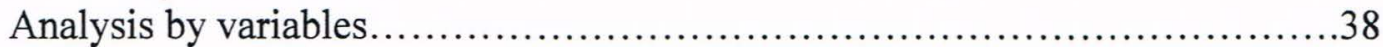

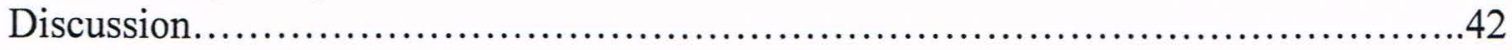

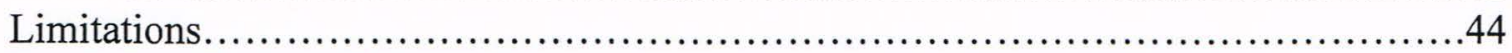

Future implications for practice................................................. 45

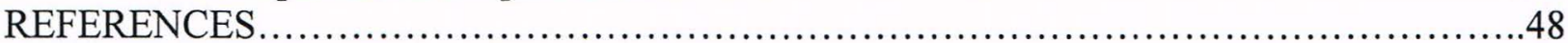

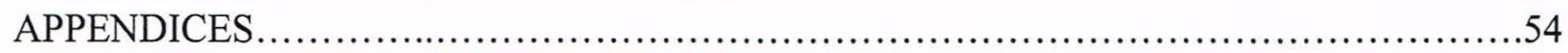

APPENDICE A: CCHCA PERMISSION LETTER FOR THE PROJECT ......................54

APPENDICE B: PERMISSION LETTER TO USE JEFFERSON SCALE OF ATTITUDE.....55

APPENDICE C:CALIFORNIA STATE UNIVERSITY, FRESNO DEPARTMENT OF

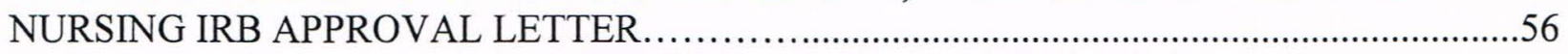

APPENDICE D: INITIAL RECRUITMENT LETTER TO PHYSICIANS ......................57

APPENDICE E: FOLLOW-UP RECRUITMENT LETTER TO PHYSICIAN ....................58

APPENDICE F: RESEARCHER-GENERATED SURVEY QUESTIONNAIRE ................59

APPENDICE G: JEFFERSON SCALE OF ATTITUDES TOWARD PHYSICIAN-APN

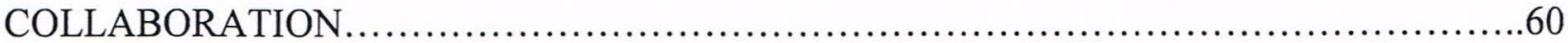




\section{LIST OF TABLES}

Table 1. Demographics of the Participants. 30

Table 2. Frequency of Responses for Researcher-generated Questions.

Table 3. Frequency of Responses for Jefferson Scale of Attitude Questionnaire $.34-36$

Table 4. Summary of Scores for the Total Jefferson Scale and Four Subscales. 37

Table 5. Reliability Test for the Total Jefferson Scale and Four Subscales......................38

Table 6. Results of Comparison Tests of Mean Total Jefferson Scores for variables...............39

Table 7. Results of T-tests for Subscales...................................................40

Table 8. Results of Spearman's Rho Correlation Test for Researcher-generated questions.......41 


\section{LIST OF FIGURES}

Page

Figure 1. Mind Map Used to Generate Survey Questions...............................21

Figure 2. Histogram of Age of Participants................................................. 31 


\section{LIST OF ABBRIVIATIONS}

APNs........ Advanced Practice Nurses

$\mathrm{CCHC}$ ..Chinese Community Healthcare Association

CRNA Certified Registered Nurse Anesthetists

IC Interprofessional Collaboration

JSAPNC Jefferson Scale of Attitudes toward Physician-Nurse Collaboration VHA Veterans Health Administration WHO World Health Organization 


\section{CHAPTER 1: INTRODUCTION}

Interprofessional collaboration has become an important component in today's national and global health care. The World Health Organization (WHO) defines that a collaborative practice happens when multiple health workers from different professional backgrounds work together with patients, families and communities to deliver the highest quality of care (Gilbert, Yan, \& Hoffman, 2010). Collaborative practice allows health workers to engage their individual skills to help achieve the patients' health goals. It also strengthens health care systems, improves health care outcomes and mitigates global health workforce crises. In addition, the WHO acknowledges that there is sufficient evidence to indicate that effective interprofessional education enables achievement of collaborative team work among health professionals (Gilbert, Yan, \& Hoffman, 2010).

Like many other developed countries, the United States has raised awareness in promoting interprofessional collaboration in an effort to address questions of quality and care in the health care system, primarily in response to patient safety and the economics of medical error. Studies have showed that the medical errors of health providers that result in serious threats to patient safety and well-being are often caused by poor quality of communication and ineffective collaboration between health providers (Haskins, 2008). Hence, improving collaboration among all health care providers is imperative to ensure safe, high-quality and costeffective patient care.

Over the past four decades, the number of advanced practiced nurses (APNs) working collaboratively with physicians has dramatically increased, and such teams provide care to countless patients in a variety of patient care settings. Within a healthcare context, APNs and physicians with diverse educational backgrounds and experiences work in common clinical 
practices to address the complex medical, social and ethical problem of the patients. The underlying relationship between the APN and physicians is the foundation of the collaborative practice (O'Brien, Martin, Heyworth, \& Meyer, 2009). Fundamental components of such collaboration often involve mutual respect and trust, regular and effective communication between team members, identification of mutual problem, shared vision and values, compatible practice philosophies and objectives, regular team education, shared decision making, adequate leadership support, as well as appropriate reward and recognition systems (Bailey, Jones, \& Way, 2005; Buppert, 2007; Clarin, 2007; Gardner, 2005; Hader, 2005; Hendel, Fish, \& Berger, 2007; O'Brien, Martin, Heyworth, \& Meyer, 2009; Sievers \& Wolf, 2006; Stein-Parbury \& Liaschenko, 2007).

\section{Problem Statement and Significance}

Until today, many studies that investigate the collaboration of nurses and physicians have shown that effective nurse-physician collaboration is associated with improved nurse retention, quality patient care, team morale, cost containment as well as satisfaction of both patients and staffs (Coeling \& Cukr, 2000; Cowan et al., 2006; Leiter \& Laschinger, 2006; Leonard, Graham, Bonacum, 2004; Lindeke \& Siekert, 2005; Phillips, Green, Fryer \& Dovey, 2001; Rosenstein, 2002; Rosenstein \& O'Daniel, 2005; Wright, 1997). Sirota (2007) acknowledges that nursephysician relationships have improved in certain settings, such as operating rooms and intensive care units, where teamwork is important, however, collaboration still seems problematic in many practices since the same conflicts between nurses and physicians that have existed for years persists today.

Although nurse-physician collaboration investigations offer insights into interdisciplinary practice teams, Almost and Laschinger (2002) state that more work is needed to 
understand the particular challenges for specifically physician-APN collaborations. APNs bring skills and knowledge unique to their roles and expect greater autonomy appropriate to their backgrounds and licensure. How is the collaborative experience of physicians in such teams working with APNs who enact roles commensurate to their training and licensure? To date, very few studies have examined the collaborative relationship between physicians and APNs (Almost \& Laschinger, 2002). Of the limited research data about their collaborations between physicians and APNs, evidences show that many barriers still exist and the major contributing factors for the poor collaboration are from professional influences such as different perspectives about patient outcomes, power struggles among team members, lack of respect, lack of understanding about the roles and scopes of practice of other providers and stereotyping with regard to other professions (Clarin, 2007; Oberle \& Tenove, 2000).

Furthermore, a review of the expansive literature within the MEDLINE, PubMed, and CINAHL databases shows that even less is known about physician-APN collaboration within an ethnic community setting, such as the Chinese community in San Francisco Bay Area, where a majority of first generation immigrants reside. Patients in this type of underserved community usually have comorbid conditions that require extensive coordinated care. The unique health behaviors of this patient population, such as language and cultural barriers, reluctance to obtain preventive care, multi-diagnostic illnesses, being late to receive care, as well as commonly used self-administration of herbal supplements and over the counter prescriptive drugs, necessitate experienced practitioners with advanced training delivering coordinated care over time. Hence, the alliance of physicians and APNs in interdisciplinary teams can be of great help to the patients in this Chinese community. Then how do physicians working in this community feel about the utilization and acceptance of APNs care? What is their common perception and attitude toward 
physician-APN collaborative practice? How similar or different are their views compared to what has been known in the literature, influenced by both Western and Chinese culture?

Undeniably, more examination is needed to assess and explore physician-APN collaboration in the ethnic communities. Understanding collaboration in such a community setting can enhance the literature on APN practice as well as enrich the knowledge about the behaviors perceived by physicians and APNs as a requisite for collaboration in the ethnic community health context. Lack of collaboration not only can lead to fragmentation of care, patient dissatisfaction and poor outcomes, but also can contribute to the role dissatisfaction and job stress for healthcare professionals responsible for insuring high quality care (Almost \& Laschinger, 2002). Assessing the perception of physicians working in the Chinese community toward physician-APN collaboration is the initial first step for promoting effective interprofessional collaboration. Exploring the facilitators and addressing the barriers using interprofessional education can be conducted consequently to endorse constructive collaboration and build an efficient interdisciplinary practice team in this unique community.

\section{Theoretical Framework}

Interprofessional collaboration has been studied by many health care providers, researchers and academicians. Both the structure and process of inter-professional collaboration have been described through several conceptual models ( D'Amour, Ferrada-Videla, Rodrigues, $\&$ Beaulieu, 2005). Most of these conceptual models for collaboration utilize the key constructs of organization theory, organizational sociology, social exchange theory and literature on team building (D’Amour et al., 1999; Giltlin, Lyons, \& Kolodner, 1994; Hayward, DeMarco, \& Lynch, 2000; Sicotte, D’Amour, \& Moreault, 2003; West, Borrill, \& Unsworth, 1998). In 
addition, two of the models are also established solely on empirical data through literature reviews (Corser, 1998; Miller, 1997).

\section{Theoretical Constructs}

There is no one theory or model found to be specifically descriptive of APN and physician collaboration. Nonetheless, the constructs and key components of these theories and models of collaboration have been utilized to describe and support nurse-physician collaboration in prior studies. Among them, social exchange theory and Corser's (1998) conceptual model of collaborative nurse-physician interactions are selected to provide a theoretical basis for this DNP project.

Social exchange theory, a middle-range theory, was first founded by the sociologist George Homans in 1958 after publishing his work named "social behavior as exchange". Homans (1958) proposed that social behavior is a result of an exchange process. He defined social exchange as the exchange of activity, tangible or intangible, and more or less rewarding or costly, between at least two persons (Homans, 1958). Homan's (1958) notion bridged a variety of disciplines and sparked differing theories of social exchange. Although different views of social exchange have emerged, theorists agree that the core idea of social exchange involves a series of interactions that overtime generate trusting, loyal relationships and mutual commitments (Mitchell \& Cropanzano, 2005).

Social exchange theory essentially is built upon several assumptions about human nature and the nature of relationships. There are two conceptualizations of social exchange: one that focuses on the nature of individuals and one that describes the relationships between two people (West \& Turner, 2004). The assumptions that social exchange theory makes about human nature include the following, 1) Humans seek rewards and avoid punishments; 2) Humans are rational 
beings; 3 ) The standards that humans use to evaluate costs and rewards vary over time and from person to person (West \& Turner, 2004). The assumptions social exchange theory makes about the nature of relationships include the following,1) Relationships are interdependent; 2) Relational life is a process (West \& Turner, 2004). Based on these conceptions, social exchange theory truly assumes that understanding social structures relies on an analysis of interpersonal transactions. Understanding the individuals' interaction is the vital element for understanding complex social behaviors between groups (D'Amour et al., 2005).

There are two fundamental concepts in social exchange theory, which are exchange and negotiation. Exchange concept implies that one will join another that provides a specific benefit and that in return, he or she must help the other individual attain his/her objectives (D'Amour et al., 2005). Exchange suggests that the reasons individuals work collaboratively in groups are to gain the benefits of group involvement. Benefits could include social support, task assistance, and professional growth, etcetera. Moreover, individuals also are expected to contribute their own skills or expertise to help reach the group goals. Subsequently, a reciprocal relationship based on exchange develops between group members. The negotiation concept refers to the process that one offers to contribute specific expertise to the other individual and in return, expects to receive specific benefits. Individuals or groups are thus constantly engaged in negotiations to try to optimize benefits, reduce costs and move forward under conditions that will be fair to all (D'Amour et al., 2005). Negotiation is also a critical element in the process of collaboration. Individuals must decide whether the benefits associated with participation of collaboration will outweigh the costs of the contributions or efforts they are expected to make. In fact, negotiation is an ongoing process that individuals and groups get involved in to ensure 
that benefits are maximized and the costs are minimized for the individuals or the team as a whole.

Homans (1958) developed five key propositions for his social exchange theory. This set of theoretical ideas represents the core of Homans's version of social exchange theory. The first proposition believes that behavior that creates positive outcomes is likely to be repeated. The second proposition suggests that the individual will continue the previous behavior if it has been rewarded in the past. The third proposition states that if the result of a behavioral action is considered valuable to the individual, it is more likely for that behavior to occur. The fourth proposition proposes that if an individual has received the same reward several times, the value of that reward will diminish. The last proposition suggests that if an individual receives more than expected, he or she will be happy and will behave approvingly (Social Exchange Theory, 1987).

Social exchange theory has been researched and studied in numerous literatures, especially in economics, psychology and sociology. It is thought to be the most influential conceptual paradigm and the best theory for understanding workplace behavior (Mitchell \& Cropanzano, 2005). Based on the philosophies of social exchange theory, the individual interaction of APN and physician are considered as interdependent and contingent on the actions of each other. APNs and physicians' interdependent transactions supposedly have the potential to generate high quality collaborative relationships in an organization. The attitudes or perceptions of physicians towards collaboration in the workplace can be examined, analyzed and understood using the key elements of social exchange theory. Subsequently, the social group behaviors and social structure of APN-physician collaboration can then be better comprehended. 


\section{Collaborative Conceptual Models}

Several models have been developed to provide a theoretical framework for team collaboration (D'Amour et al., 1999; Giltlin, Lyons, \& Kolodner, 1994; Hayward, DeMarco, \& Lynch, 2000; Sicotte, D'Amour, \& Moreault, 2003; West, Borrill, \& Unsworth, 1998). Two of them, by West, Borrill and Unsworth (1998) and Sicotte, D'Amour and Moreault (2003), are derived from organizational theory and one model is based on organizational sociology (D'Amour et al., 1999; 2004). These models are thought to be less relevant to assist understanding of APN-physician collaboration since these models are often used to test for team effectiveness and evaluate inter-organizational and intra-organizational collaboration. Although these are two models based on social exchange theory (Gitlin, Lyons \& Kolodner, 1994; Hayward, DeMarco \& Lynch, 2000), they both are focused on the developmental process of collaboration and did not illustrate the structures and critical attributes of the collaboration. As a result, they are less valuable in guiding the analysis of perceptions toward APN-physician collaboration.

The Corser's (1998) conceptual model of collaborative nurse-physician interactions was created to comprehensively illustrate the salient variables that have been suggested in the professional literature as affecting the quality, rate, and outcomes of the fundamental component of nurse-physician collaboration. Although this model is not directly originated from social exchange theory, it refers to these same concepts, just express them in different ways. In addition, the relationships and interactions of APNs and physicians closely resemble that of nurses and physicians given the historical background of the two professional groups.

Consequently, Corser's (1998) model is determined to be an appropriate and better fit as the theoretical framework to guide the APN-physician collaboration project. 
The Corser's (1998) model established taking into account the structures and processes of collaboration. It was largely derived from empirical data from the literatures that have attempted to more clearly specify the factors that appear to most frequently affect the occurrence of collaborative interactions between nurses and physicians (Corser, 1998). The model is based on the assumptions that the fundamental unit of most collaborative work relationships is the collaborative interaction, an exchange that will be generally established through direct face-to face, phone, e-mail, and text messages periodically between the nurse and physician. Corser (1998) suggested that nurse-physician collaboration involves both personal and interpersonal influences, as well as the organizational and professional influences. A genuine collaborative relationship requires mutual respect for each other's' professional roles; it also requires that both the nurse and the physician maintain actual and perceived power symmetry with respect to each other. The most important outcome of collaborative interactions is a more consistent achievement of clinical patient goals. Furthermore, Corser (1998) asserted that the collaborative interactions may often be subtly influenced by forces that neither the individual nurse nor physician may typically appreciate. The collaborative interactions will not occur unless both the nurse and physician practice in settings that are supportive of such exchanges and have been educated professionally, socialized and are personally inclined to demonstrate collaborative behaviors as they work with each other. Corser's (1998) model has been tested to guide a case study on collaboration among nurse practitioners and registered nurses in outpatient oncology settings (Moore \& Prentice, 2013).

Collaboration needs to be understood not only as a professional endeavor, but also as a human process. Social exchange theory and Corser's (1998) model certainly offer the theoretical framework on understanding the why and the how of collaborative activities was perceived 
among humans. They both identified that collaborative behavior has an exchange factor, as well as analyzed the main concepts of personal/interpersonal influences and organizational/professional influences that affect sharing, partnership, interdependency and power struggle related to collaboration using their distinctive approaches. Although these theoretical frameworks have their own limitations, they provide researchers with a basic notion to understand collaborative practice. These fundamental conceptions, therefore, are used to guide the generation and selection of the survey questions in this capstone project. In the future, it can also be used to diagnose the degree of collaboration achieved and to identify areas for improvement in a target clinic or an organization. 


\section{CHAPTER 2: REVIEW OF LITERATURE}

A literature review was conducted by searching for articles from Databases, which include the Cochrane Library, the Joanna Briggs Institute Library, PubMed, Medline, CINAHL, and ProQuest. Numerous researches identified investigated the physician-nurse collaboration. The literature search related to the topic of collaboration between APNs and physicians in all clinical settings reveals a few qualitative and quantitative descriptive studies. Moreover, there is no literature concerning physician-APN collaboration specifically within a Chinese community environment. Within the limited studies that describe physician-APN collaboration, the subjects can be categorized into three areas: attitudes about collaboration, barriers to collaboration, and interprofessional collaboration education. The focus of this literature review consequently will be on the research that focused on assessing physicians' attitudes toward APNs collaboration.

A recently published integrative review, which used systematic review processes, was undertaken to summarize qualitative and quantitative studies published between 1990 and 2012, in regards to the perceptions of physicians and APNs on collaborative practice in primary health care settings (Schadewaldt, McInnes, Hiller, \& Gardner, 2013). The findings of this integrative review reflect the general ideas of the overall body of literature that describes physician-APN collaboration. Twenty-seven studies conducted in seven different countries met the inclusion criteria. All of the studies that met the inclusion criteria were assessed for quality. Content analysis identified a number of barriers and facilitators to collaboration between physicians and APNs. Five themes were developed in relation to perceptions and understanding of collaboration. Physicians and APNs have differing views on the essentials of collaboration and on supervision and autonomous nurse practitioner practice. Physicians who have a working experience with APNs express more positive attitudes towards collaboration. Both professional 
groups report concerns and negative experiences with collaborative practice but also value certain advantages of collaboration (Schadewaldt, McInnes, Hiller, \& Gardner, 2013).

This is the first systematic review to specifically look at APNs, not general nurses' experience and view of working collaboratively with physicians in primary health care. Their findings are informative, comprehensive and reliable. The limitation of the review is that there is no secondary reviewer assisted in the appraisal of studies and extraction of data. Not all the qualitative studies have reported the researcher's background. In addition, although all included studies examined APNs who were educated at a postgraduate degree level and who practiced at an advanced level that included the diagnosing of patients, regulations around the APN role, licensure and practice vary among and within countries. Therefore, themes and factors identified in this review may only apply to the particular APN role in the primary health care setting of the country of the study.

Within the limited articles exploring specifically the attitudes toward physician-APN collaboration, there are a few articles that warrant mentioning. Zander (2005) initiated a descriptive comparative study for her doctoral dissertation, focused on attitudes toward collaborative practice of physicians and APNs from within the state of Florida. Data was gathered using an online survey for APNs and physicians who used the researcher's modified version of the Jefferson Scale of Attitudes Toward Physician/APN Collaboration, designed to explore physicians' and APNs' attitudes toward collaboration (Hojat \& Herman, 1985). Overall, APNs $(n=64)$ had more positive attitudes on the modified Jefferson Scale than physicians $(n=$ 9). The biggest limitation of this study was that the participants' response rate was very low, even though the study randomly surveyed 400 APNs and their supervising physicians but the 
number of participating physicians still differed radically from the number of participating APNs.

In the study of Jones and Fitzpatrick (2009), a sample of Certified Registered Nurse Anesthetists (CRNA) and anesthesiologists affiliated with postgraduate training programs in the state of Texas, responded to a survey designed to gather attitudes toward CRNA-physician collaboration using an adaptation of the Jefferson Scale of Attitudes Toward Physician-Nurse Collaboration. Sixty two anesthesiologists and two hundred and eight CRNAs completed the survey. The mean for the total scores on attitudes toward collaboration for anesthesiologists was 44.4 (+/- SD 8.7) and 51.8 (+/- SD 2.7) for CRNAs. This indicates that the attitudes for CRNAs were consistently higher in the 4 subscales of collaboration than for anesthesiologists. No significant differences in attitudes were found between men and women for the total sample. The study also suggests that CRNAs who deal with role conflict or unclear expectations as well as limited scope of practice may have increased job stress and dissatisfaction. The samples sizes from both CRNAs and physicians in this study were rather large, which produced more reliable findings. However, the sample sizes of two groups were still not homogenous with the response rate of physicians continuing to be low.

Fletcher et al. (2007) described APNs' and physicians' perceptions of the role of APNs, the degree of collegiality between professions, and the APNs' feeling of acceptance as the primary care provider. This descriptive study included both closed- and open-ended questions plus several Likert-type questions. The sample was all primary care APNs (87) and physicians (162) within a Midwestern Veterans Health Administration (VHA) region. Data were collected from 153 providers. APNs saw their role as one of autonomous practice with physician back-up as needed, while physician respondents envisioned a role akin to a physician extender. Most of 
the physician respondents did not think APNs could provide adequate primary care to veterans who tend to have many comorbid conditions. Yet both groups considered their relationships to be collegial and most APNs felt accepted by physicians. Physicians particularly valued APNs' teaching and interpersonal skills leading to greater patient satisfaction. This study had an overall good response rate of $61.4 \%$ and data validation through mixed methods questionnaire. The limitations of the study included the fact that the participant selection process was unclear and no psychometric properties of the questionnaire were reported.

Street and Cossman (2010) analyzed how physician characteristics and close working relationships with APNs influence physicians' attitudes toward APNs. Using 463 Mississippi Physician Workforce Study survey data, the study identified physician characteristics associated with having APNs in practices and discrete APN-attitudinal items. Generalists, physicians in public sector employment and physicians in larger practices are more likely to work in practices that also include APNs. Physicians working with APNs are somewhat younger than those who do not. Regression analysis indicates that male physicians had less-positive attitudes toward APNs, while physicians who practice alongside APNs and who have been in practice longer have the most positive generalized attitudes toward APNs. Physicians who work in the same practice with APNs have more positive attitudes toward them. However, regardless of work arrangements, physicians are reluctant to let APNs to practice independently. Physicians with early collaborative training with APNs may have more positive attitudes, but even such exposure will not necessarily lead physicians to support APNs' independent practice. The strength of the study was that it had a large sample size and it also used a validated questionnaire with Likert scales as the assessment tool. The limitation of the study was that it had a low response rate of $23.3 \%$ and a convenience sampling method limits the study finding's generalizability. 
In short, the evidence shows that physician-APN collaboration is a slow progression. Physicians and APNs face a number of barriers when working in collaboration. Generally, physicians tend to rate their collaborative practice experience lower than APNs. Exposure to working together seems to help to overcome professional hurdles, dispel concerns and provide clarity around roles and the meaning of collaboration of physicians and APNs. However, no data found to describe the physician-APN collaboration view and experiences in an ethnic community. Questions remain unanswered: What do physicians working in a Chinese community feel about working with APNs in their practices? How is their perception on physician-APN collaboration? Are their experiences and attitudes similar or different to what has been known in the literature? Further exploration on this topic is needed and would be useful to generate insightful knowledge and attempt to fill the gap in the literature. 


\section{CHAPTER 3: METHODOLOGY}

This quantitative descriptive capstone project focused on assessing the general perception among physicians, who work in the Chinese Community of the San Francisco Bay Area, toward collaborative practice with nurse practitioners. Data was gathered using a survey tool consisting of six researcher self-generated 5-point Licker-scale questions and a modified 15-item Lickertype instrument entitled, the Jefferson Scale of Attitudes toward Physician/APN collaboration (Hojat \& Herman, 1985). The specific aims of this study are to explore

- The common perception of physicians toward collaboration with APNs.

- The physicians' culture beliefs about harmony and collaboration

- The organizational influence on APN utilization in the physicians' current practice settings

- The physicians' perception of Chinese patients' acceptance of APNs' care.

- The willingness of the physicians to hire and work with APNs.

This study serves as an initial step of an organizational quality improvement project. Based on the findings of this study, areas of deficiency and competence of inter-professional collaboration can be identified. Education and training thus can be provided to enhance interprofessional collaboration within the organization. Consequently, the ultimate goals of quality improvement, clinicians' and patients' satisfaction can be achieved.

\section{Settings/Organizational Structures}

This study is conducted in the Chinese Community Health Care Association (CCHCA). CCHCA is a medical group founded in 1982. It's specific and primary purpose is to promote social welfare by making health services more accessible to the Chinese community of San Francisco and the Bay Area. The CCHCA is a non-profit tax-exempt association. The 
membership of CCHCA is composed of members of the Chinese Hospital medical staff and CCHCA San Mateo Affiliate physician members, who are not on the Chinese Hospital medical staff, but their primary office is located in San Mateo County and they have privileges at Seton Medical Center, or at an acute hospital affiliated with CCHCA.

An elected Board of Trustees of eleven members plus one appointee from the Chinese Community Health Plan (CCHP) manages the affairs of CCHCA. There is also an advisory board of non-physicians which meets three times a year and has two representatives on the Board of Trustees. CCHCA has several standing committees, including a compensation committee, finance committee, grants program committee, membership committee, nominating committee and quality assessment/utilization review committee. Committee members are appointed by the Board of Trustees.

The majority of the physicians in the CCHCA work in a small group or an individual private practice, while another small portion of the physicians work in support health clinics that are affiliated with Chinese Hospital. From the macro-level of the association to the micro-level of each individual physician's office, the existing leadership and innovation of the Chinese community is influenced by the traditional Confucian culture and organizational ethical climate to an extent. Being different from the Western concept of "relationship," the Chinese concept of "relationship," that is, "guanxi" profoundly influences Chinese society in commercial activities, business ethics, and organizational behaviors (Lin, 2011). Moreover, business, such as the medical offices, can develop their networks of guanxi to gain competitive advantages (Lin, 2011). Indeed, CCHCA is a social network system that allows a group of ChineseAmerican physicians working together to gain professional and financial advantages and better 
compensation in the healthcare system. Creating a good, harmonious relationship or "guanxi" with the leaders and physicians in the organization is essential for any proposal implementation. Creating an innovation also requires an understanding of the leadership structure in the organization (Porter-O'Grady \& Malloch, 2011). The board of trustees in the CCHCA, acting as the executive team of the organization, manages operational affairs and provides access, linkage and evaluation of any innovative proposal affecting the organization. The board members consist of a diverse group of physicians that analyze information gathered through first-hand, first-person experience and deliver unique views on issues or proposals that impact the organization and the community. The innovative ideas that are in line with the interests and goals of the CCHCA tend to be approved easily and supported thoughtfully by the board members.

Although the board of trustees has certain executive powers and authorities for controlling and administering components of the organization, $\mathrm{CCHCA}$ 's decisional structure is mainly a horizontal relationship since all the physician members are partners in this professional community. As a result, engaging the physicians in this innovative research is vital for transforming the research evidence to practice. As Porter-O'Grady and Malloch (2011) stated, exploring and clarifying the expectation of members' roles in an innovative organization are critical once the agreement and role expectation of leaders are well-established. Understanding the innovativeness of the CCHCA physicians is imperative for the implementation of this project. It is undeniable that the perspectives of the CCHCA physicians on innovation are impacted by two interacting cultures- the U.S. culture and the Chinese Culture. It is generally found that Western cultures (U.S. culture), tend to be more innovative than Eastern cultures (Chinese Culture), in this case (Wong, Everett, \& Nicholson, 2008). Chinese-American leaders 
and physicians have a high potential to be more innovative than the ones from the Chinese Culture, and may or may not be less innovative than the ones educated solely under the Western culture (Wong, Everett, \& Nicholson, 2008). Frequent empowerment and reinforcement are necessary to increase the participation and awareness of the physicians in this study. Key strategies to engage the physicians are to align the goals of this study with their perspectives and values in their practice. In addition, it is anticipated that future innovative changes may occur slowly in this group given their unique cultural characteristics. Consequently, more effort is required for innovation implementation in this organization and community.

\section{Participants}

This study uses the convenience sampling method. Two hundred seventeen physicians, who are members of the Chinese Community Health Care Association (CCHCA), are the target participants in this study. The majority of the members in CCHCA are Chinese-American physicians. Most of them have a doctorate degree in medicine (MD) and a few of them are doctorates in osteopathic medicine (DO). Given the nature of the study, the members who are non-physician clinicians, such as dentists, psychologist and podiatrist are excluded from the recruitment since they are unlikely to work with APNs. Certainly, physicians who do not belong to the CCHCA are excluded from this study.

\section{Recruitment Procedures}

The name and address labels of 217 member physicians were obtained from the operation director of CCHCA. Each label was then placed on a large white envelope by the researcher in preparation for mailing. Inside of each white envelope was a study introduction letter, a researcher generated survey questionnaire, a modified Jefferson Scale of attitudes toward physician/APN collaboration, and a returning envelope with the researcher's address and a 
prepaid postage. The introduction letter explained the name and the purpose of the study, intended to recruit the participants. Confidentiality issues were addressed in the introduction letter (see Appendix D). The physicians were informed that their participation in the study is voluntary and anonymous. By returning the survey questionnaire, the physician implied informed consent for participating in the study. In the end, the researcher's personal email address was made available as the contact information for the physicians who want to know the study results in the future. The final study results will be shared with the leadership team of the CCHCA in order to conduct potential future educational training courses, aiming to improve inter-professional collaboration patterns within the association.

\section{Instruments}

The survey questionnaires, composed of two parts, were utilized as the instrumental tool in this study. The first part of the survey included demographic inquiries and a six 5-point Likert-type scale questions created by the co-investigator (see Appendix F). The first three questions were intended to evaluate the personal, cultural, and organizational influential factors on the participants' attitude toward collaboration. These factors are the illustrated influences based on Corser's (1998) conceptual model of collaborative which served as the study's theoretical framework. The remaining three questions were to assess patients' and physicians' acceptance toward APN care and the physicians' willingness to hire or work with APNs in the future if their practices expand. These questions were thought to be informative and important to for the CCHCA leadership to determine the benefits of APN utilization in the organization for the near future. The completed six 5-point Likert-type scale questions created by the coinvestigator (see Appendix F) were reviewed and checked for content accuracy and fluidity by 
the two concept experts Dr. Ginny Fong and nurse practitioner Donna Chee. Figure 1 illustrated the mind map used to generate the questionnaire.

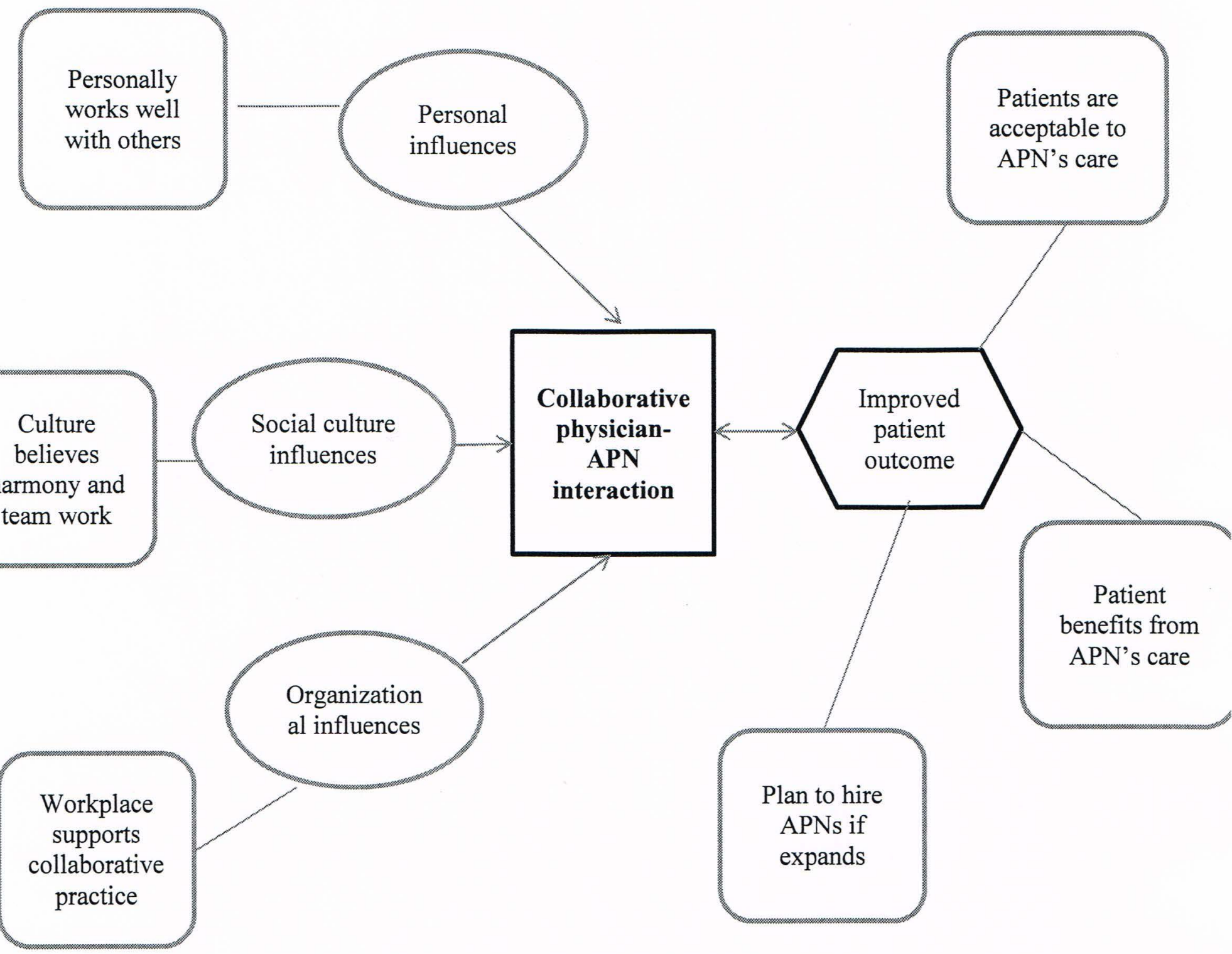

Figure 1: Mind Map Used to Generate Survey Questions 
The second part of the survey questionnaires was the modified version of the Jefferson Scale of Attitudes toward Physician/APN collaboration (see appendix G), which measures physician's attitudes toward authority, autonomy, responsibility for patient-monitoring, collaborative decision-making, role expectations, and collaborative education. Permission was obtained from the author to use this instrument and modify the original questions in the Jefferson scale of Attitudes Toward physician/nurse collaboration (JSAPNC) in adapting to this study needs (see Appendix B). This instrument was based on the rationale that inter-professional collaboration is a joint venture, with shared authority and responsibility, open communication, and shared decision-making. The education of professionals within a collaborative environment would also affect the attitude of nurses and physicians toward each other and the concept of collaboration (Dougherty \& Larson, 2005).

There were 15 questions that were answered on a 4-point Likert-type scale in the JSAPNC instrument. Question items were categorized into four subscales that correlate to four factors contributing to inter-professional collaboration. Items $1,3,6,9,12,14$, and 15 belonged to the subscale of shared education and collaboration; items 2, 4, 7 were for the subscale of caring versus curing; items $5,11,13$ were to assess APN's autonomy and items 8,10 were to evaluate physician's authority (Hojat et al., 1999). Scoring was accomplished by determining if an item on the scale reflected either a positive or negative attitude toward physician/APN collaboration. Most of the items were directly scored based on their Likert weights (Strongly agree $=4$, agree $=3$, disagree $=2$, strongly disagree-1). However, items 8 and 10 were reverse scored items (strongly agree $=1$, agree $=2$, disagree $=3$, strongly disagree $=4$ ). Total score was the sum of all item scores . The higher score was an indication of the more positive attitudes toward physician-APN collaboration (Hojat et al., 1999). 
The JSAPNC instrument can be used for both physicians and nurses. Hojat et al. (1999) determined content and construct validity of the JSAPNC by comparing consistency of constructs with conceptual discussion of physician/nurse professional collaborative relationships found in the existing literature. This instrument was initially tested on first-year medical students and upper-division baccalaureate nursing students. Internal consistency estimates of reliability or Cronbach's alpha was 0.84 for medical students and 0.85 for nursing students. The item total score correlations for the combined group ranged from 0.65 to 0.40 with a median correlation of 0.61 (Dougherty \& Larson, 2005). Later, this instrument's validity and reliability was confirmed in a study with 333 nursing students with a reliability coefficient of 0.77 . Thus, this psychometrically sound instrument can be used with confidence to empirically examine attitudes toward inter-professional collaboration in a variety of settings.

Although the use of students with limited practice experience to test the validity and reliability of the instrument may limit its utilization with practicing nurses and physicians, Hojat et al. (1999) suggested the JSAPNC could be exploited in studies involving different specialties and sub-specialties to explore gender, age, ethnicity differences and geographical locations. Hojat et al. (1999) also recommended this instrument as a research tool for studies with professional practicing nurses and physicians. Since then, the modified version of JSAPNC has been developed in a few prior studies that examined varies of physician-APN collaborative relationship (Jones \& Fitzpatrick, 2009; Zander, 2005). Hence, the application of the modified version of the JSAPNC in physician-APN collaborative studies will most likely achieve similar validity and reliability. Moreover, the JSAPNC has been used in study that primarily to measure the attitude of nurses and physicians in different cultures and countries toward the concept of collaboration (Hojat et al., 2001; Hojat et al., 2003). But it has never been tested in an Asian 
culture group. This capstone project will be the first attempt that used the JSAPNC instrument to identify attitudes toward physician/APN collaboration in a Chinese community.

To better fit the study purpose of this capstone project, the original JSAPNC scale was modified as described in below. First, in the modified version of JSAPNC, the word "nurse" was replaced by "APN" throughout the survey. Secondly, question items were re-categorized to fit the four subscales that correlate to four factors contributing to inter-professional collaboration based on its appropriateness. Items $1,3,6,9,14$, and 15 were set to the subscale of shared education and collaboration. Items 2 and 7 were placed to the subscale of caring versus curing. Items $4,5,11,12$, and13 were used to assess APN's autonomy and items 8 and10 were kept in the same last category to evaluate physician's authority.

\section{Human Subjects}

Permission for conducting the study was first obtained from the CCHCA director in April, 2014 (see Appendix A). It was then approved by the Institutional Review Board of the California State University, Fresno in June, 2014 (see Appendix C). The investigator followed policies and procedures for the protections of human subjects. An introduction letter informed the participants about the investigators, the study, its purpose and the participants' rights. By returning the survey via traditional mail or electronic web link, the participants implied consenting for the study.

Overall, potential risks associated with participation in the study were unlikely and of low risk. There was little likelihood of any physical risk as a result of participation in this project, since the participants were not asked to perform any tasks that could result in physical harm. Participants were asked to provide information about their attitude and opinions related to physician-APN collaborative practices as well as their demographic and personal data (age, 
gender, specialty, and ethnicity). These questions had a small likelihood of low psychological risk if participants were upset by questions that ask them to think about their experiences and feelings about physician-APN collaboration in their practices that may have been disturbing. The social risks associated with this study were small. In general, bringing the social and professional issues to a conscious level has a considerable likelihood of mild to moderate social risk in terms of conflicts with cultural, professional beliefs, traditions, and social order. The investigator proposed that this risk could be managed and channeled in a positive way through careful attention and training which would potentially be a part of the intervention after this project. Additionally, there may be a perceived risk among physicians that participation in this project may impact their relationship with APNs or other support health alliances in a negative way. Member physicians may perceive a risk that participation in this project may impact the quality or existence of support services they receive (currently or in the future) from the CCHCA.

In order to minimize the risks, participants were informed that they are free to refuse to respond to any question that may result in psychological disturbance. Written information was collected for research purposes only and it did not become part of the CCHCA member physicians' personnel records. Individual responses to the research questionnaire was not linked to identifying information and was not at all influencing current or future receipt of services from the CCHCA. These precautions were expected to be completely effective in reducing risks associated with participation. Confidentiality was maintained at all times. The mailed surveys were completed by the participants and were returned to the co-investigator in a sealed envelope without any identifiable information. The web link to the surveys created in SurveyMonkey was attached to CCHCA's online newsletter by the CCHCA staff member, so the investigators were 
not able to recognize the participants. Similarly, the web-based surveys also did not require any private information or log-in code so that the participants' identities were protected. The original returned surveys were stored with the co-investigator at her home in a secure locked location until they were categorized and statistically analyzed.

\section{Data Collection}

Both mailed surveys and web-based surveys were used for data collection from July 1, 2014 to October 31, 2014. Mailed surveys were sent out to all the target participants first in July, 2014. The mailed surveys were served as the primary method for data collection. In September 2014, the same survey, computed in SurveyMonkey website went out to the same physician group with the CCHCA's monthly online newsletter. The web-based surveys were served as the follow-up method and intended to catch more computer savvy participants in a relatively shorter time. The surveys link on SurveyMonkey website kept active for two months to allow sufficient time for data collection.

Kaplowitz, Hadlock, \& Levine (2004) conducted a survey mode comparison study (N = 19,890 ) and found that a mailed survey and a web-based survey application can achieve a comparable similar response rate, with the higher response rate (about $31 \%$ ) by traditional mail communications \& surveys, and the lower response rate (about $21 \%$ ) by e-mail communications \& surveys. Based on this information, the mailed survey was chosen to be the primary data collection method for the study. By the end of August 2014, there were 33 returned surveys received and by the end of the study on October 31,2014, 11 more surveys were returned by mail.

In order to increase the response rate, a follow-up letter was posted on the CCHCA's September online newsletter to re-introduce the study and encourage the physicians who have 
not yet participate via the mailed survey previously to answer the same surveys electronically. A web link to the survey that generated on SurveyMonkey website was attached below the followup letter on the newsletter. The web link would take the participants to the survey directly without providing any sign-in or access code or identifiable information. However, no physician responded to the online survey by the end of October 2014. The co-investigator then decided to close the web survey link on October 31, 2014 due to its inactivity in the past two months. Webbased survey was determined as an ineffective method for data collection in this study. The reasons for that might be multifactorial. One suspected reason might be that the physicians did not even open the online newsletter because they were too busy or not familiar with electronic mails, so they did not even see the follow-up letter and the web link for the survey. Or the physicians felt too much trouble to go to another website (SurveyMonkey) to answer the survey online. Another possible reason was that the physicians who haven't returned the mailed survey were not interested in the study anyway, so they chose not to respond no matter the reminder was by mail or online.

Due to the time constrain of the study, the co-investigator did not have time to send out another follow-up letter to recruit more participants and data. The response rate of this study, therefore, was $20.3 \%$, based on 44 mailed responses out of 217 targeted physicians. According to a synthesis review of survey response rates in organizational research, the average response rate for studies that utilized data collected from individuals was 52.7 percent with a standard deviation of 20.4, while the average response rate for studies that utilized data collected from organizations was 35.7 percent with a standard deviation of 18.8 (Baruch \& Holtom, 2008). The low response rate of this study, therefore, was within normal trend as expected. 


\section{Data Analysis}

Data were inputted by hand into IBM SPSS Statistics Version 22 and descriptive statistics were computed for the sample characteristics using frequency, percentages, mean, range and standard deviation as appropriate to the measurement level of each variable. The common pattern from the independent variables (gender, ethnicity, age and past experience with APNs) and their relationship with dependent variables (6 investigator-generated questions and JSAPN scores) were evocatively analyzed. The participants' JSAPNC group mean was also descriptively compared to the known mean score for the American physicians (M 48+/- SD 4.9) based on the existing study (Hojat et al., 2003) in attempt to gauge the knowledge of attitudes of Chinese physicians.

In this section, the design of the study, the instrument used and the sample selection were carefully described. The method of data collection and analysis were also discussed in depth. Furthermore, a few limitations to the study, such as small sample size and low response rate of survey, were identified. In the following final chapter, the results of the study are reported. It concludes the ultimate study findings and recommendations for future research on the topic. 


\section{CHAPTER 4: RESULTS AND DISCUSSION}

This descriptive study focused on assessing the physicians' attitudes toward collaboration with APNs within the Chinese Community Health Care Association. It attempted to understand the Chinese physicians' perception on this issue and hoped to discover whether their cultural or ethnic background affected their attitudes toward collaborative practice with APNs. The study was conducted over a 4-month period. A total of 217 surveys were distributed to the Chinese Community Health Care Association physicians and 44 (20\%) were returned. The follow-up survey was also sent to the 217 physicians electronically via their email address that was linked to the association's newsletter distribution lists. No electronic version of the survey was returned by the end of the study time frame. Thus, the overall return rate of the study remained at $20 \%$.

\section{Demographics}

Table 1 shows the demographic information of participants. Majority of the participating physicians $(94 \%$; $n=41)$ were Asian, of which $90 \%(n=39)$ were Chinese. The rest of the participants identified themselves as Caucasians $(n=2)$ and other $(n=1)$. This unique distribution of ethnicity closely resembles the true physician ethnicity composition of the CCHCA, which indeed near $90 \%$ of the physicians are Chinese and serve mainly the first or $2^{\text {nd }}$ generation Chinese immigrants in the San Francisco Bay Area. Almost two thirds of the participating physicians were male $(66 \% ; n=29)$, and the other one third of them were female $(34 \%, n=15)$.

Physicians were asked to report their primary specialty in their current practice. The results showed that the frequency distribution of the respondents was family medicine (9\%), internal medicine (16\%), OBGYN (16\%), pediatric medicine (11\%) and other specialty (48\%). Since family medicine, internal medicine, OBGYN and pediatric medicine are commonly viewed as primary care providers, the respondents consequently were grouped into two sets for 
easy analysis purpose: primary care (52\%) and specialists (48\%). Incidentally, the number of the participating physicians in each group is close to equal

The finding also revealed that majority of participating physicians in this study had been or were currently working with APNs $(70 \%, \mathrm{n}=31)$ in their medical practices. Only $30 \%$ of them reported that they have never worked with APNs ( $\mathrm{n}=13)$.

Table 1.

Demographics of the Participants

\begin{tabular}{lcc}
\hline Variable & Frequency & Percent \\
\hline Ethnicity & 39 & 90 \\
$\quad$ Chinese & 2 & 4 \\
$\quad$ Non-Chinese Asian & 2 & 4 \\
White & 1 & 2 \\
$\quad$ Others & & \\
Gender & 29 & 66 \\
$\quad$ Male & 15 & 34 \\
Female & & \\
Specialty & 4 & 9 \\
Family Medicine & 7 & 16 \\
Internal Medicine & 7 & 16 \\
OBGYN & 5 & 11 \\
Pediatric Medicine & 21 & 48 \\
Other Specialists & & 70 \\
Prior Working Experience with & 31 & 30 \\
APNs & 13 & 100.0 \\
$\quad$ Yes & & \\
No & 44 & Total
\end{tabular}

The frequency distribution of age of the participants is demonstrated by the histogram showed on Figure 2. Ages were spread broadly in the age continuous spectrum, with the youngest physician was at 30 years old and the oldest one was at 76 years old. More than half of the participating physicians (64\%) were in the $40-50$ year old range. The mean age of the physicians was 48.55 years $(\mathrm{SD}=+/-10.48)$. 


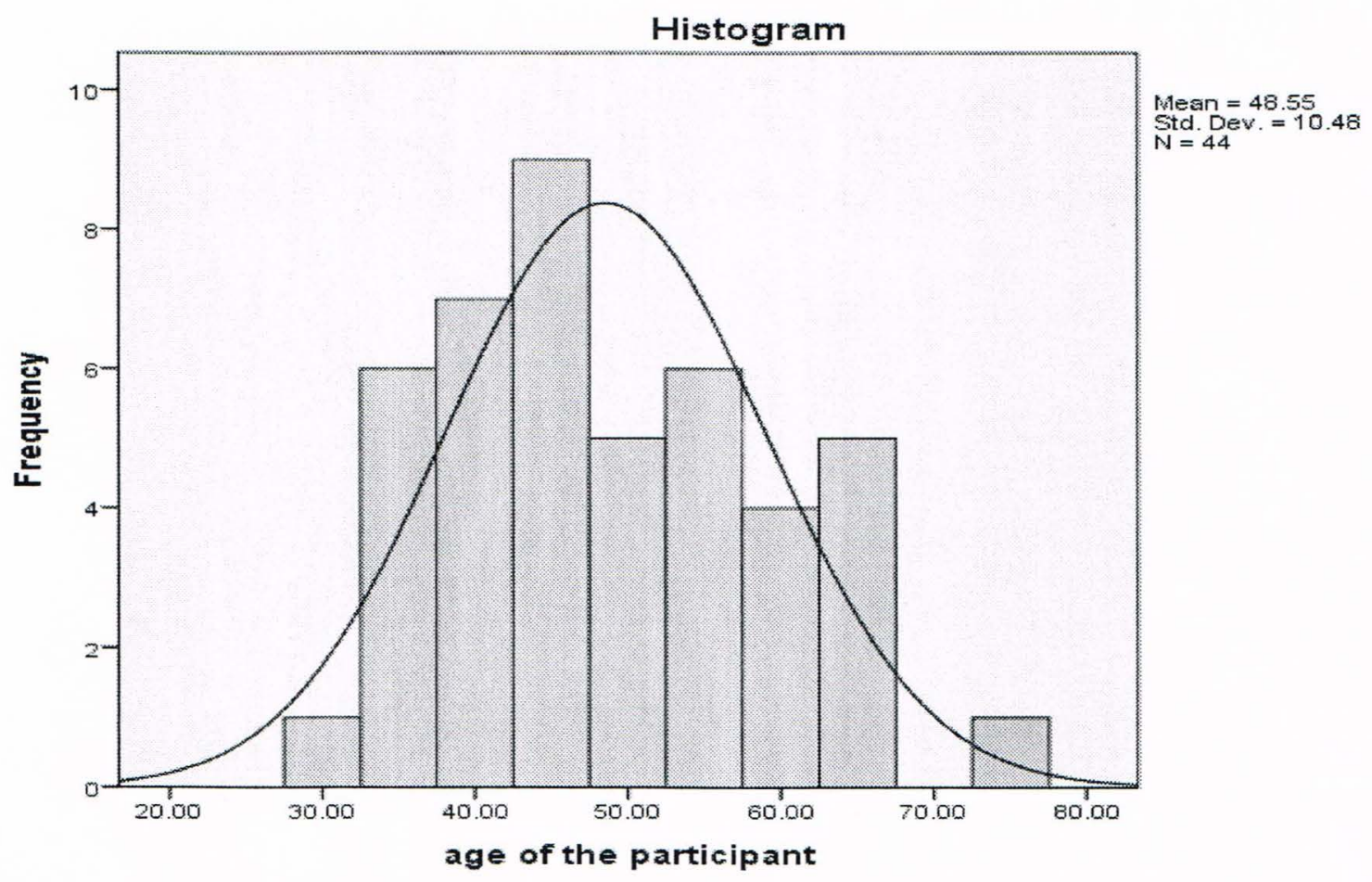

Figure 2. Histogram of Age of participants

\section{Researcher-generated Questions Analysis}

All respondents completed the six researcher self-generated questions (see appendix F).

As described in the methodology chapter, these questions were intended to evaluate the personal, cultural, and organizational influential factors on the participants' attitude toward collaboration, as well as to assess physicians' perception on acceptance and benefits of APN care in their current practice. These questions were developed in addition to the Jefferson Scale questionnaire, hoping to obtain more relevant information for the CCHCA leadership in order to determine the benefits of APN utilization in the organization for the near future.

Table 2 shows the percentage of the responses for each question. $98 \%$ of participating physicians agreed or strongly agreed that they work well with others and $82 \%$ of them believed that their culture promotes harmony and team work. At least $68 \%$ of the participating physicians felt that their office/organization had supportive utilization of APNs. $63 \%$ of them believed that 
the patients are acceptable to the APNs' care and most of them (77\%) thought that the patients can benefit from APNs' care. However, half of the participating physicians (52\%) responded that they might consider hiring APNs if their practice expands.

Table 2

Frequency of Responses for Researcher-generated Questions

Question item Percent

1. I work well with my colleagues and support staff.

Strongly Disagree Percent

Disagree

Neutral

Agree

30

Strongly Agree

68

2. My culture promotes harmony and team work.

Strongly Disagree $\quad 0$

Disagree $\quad 2$

Neutral $\quad 16$

Agree $\quad 32$

Strongly Agree $\quad 50$

3. My office/organization supports utilization of mid-level practitioners such as APNs.

Strongly Disagree

Disagree

4

Neutral

Agree

Strongly Agree

4. I believe my patients are acceptable to APNs' care.

Strongly Disagree

Disagree

0

Neutral

Agree

Strongly Agree

5. I believe my patients can benefit from APNs' care.

Strongly Disagree 2

Disagree $\quad 2$

Neutral $\quad 18$

Agree $\quad 48$

Strongly Agree $\quad 30$

6. I plan to hire or work with APNs if my practice expands.

Strongly Disagree

Disagree

Neutral

Agree 


\section{Jefferson Scale of Attitudes Analysis}

\section{Results of Jefferson scale survey}

All participants filled out the modified version of the Jefferson Scale of Attitudes toward Physician/APN collaboration (JSAPNC) (see Appendix G). As previously mentioned, the JSAPNC questionnaire is the key instrument of this study. It measures physician's attitudes toward authority, autonomy, responsibility for patient-monitoring, collaborative decisionmaking, role expectations, and collaborative education. As a result, the scale scores reflect their insight towards collaboration with APNs.

Table 3 shows the frequency and percentage of responses for the 15 JSAPNC items. For the purpose of easy interpretation, the responses were examined by agree versus disagree options, after collapsing the answer of the strongly and tend to agree together and the strongly and tend to disagree together. Three quarters of physicians agreed that an APN should be viewed as a collaborator and colleague. The majority of physicians felt that APNs are qualified to assess and respond to psychological needs of patients.

Moreover, all of the physicians thought that medical and nursing students should be involved in teamwork during their education. And more than $90 \%$ of physicians agreed that APNs should be involved in making policy decisions affecting their working conditions and all of them said that APNs should be accountable for their care provided. In addition, the majority of physicians stated that there were many overlapping areas of responsibility between them and the APNs and that the APNs actually have special expertise in patient education and psychological counseling. Although there were less than half of the physicians that believed the primary function of the APN is to carry out the physicians' orders, three quarters of them still said that doctors should be the dominant authority in all health care matters. 
On the other hand, more than $90 \%$ of physicians claimed that both APNs and physicians should contribute to decisions regarding hospital discharge of patients and most of them felt that APNs should be involved in policy decision making concerning the hospital support services. Furthermore, all of the physicians agreed that APNs should have responsibility for monitoring the effects of medical treatment and they should question a physician's order if they feel that it might have the potential for detrimental effects on the patient. Lastly, less than $10 \%$ of the physicians believed that they should not be educated to establish collaborative relationships with APNs and that the interprofessional relationships education is not beneficial and should not be included in their educational programs.

\section{Table 3}

Frequency of Responses for Jefferson Scale of Attitude Questionnaire

Question item Percent (\%)

1. An APN should be viewed as a collaborator and colleague with a physician rather than his/her assistant

Strongly Disagree $\quad 4$

Tend to Disagree $\quad 21$

Tend to Agree $\quad 39$

Strongly Agree 36

2. APNs are qualified to assess and respond to psychological aspects of patients' needs

Tend to Disagree $\quad 0$

Tend to Disagree $\quad 9$

Tend to Agree $\quad 45$

Strongly Agree 46

3. During their education, medical and nursing students should be involved in teamwork in order to understand their respective roles

Tend to Disagree $\quad 0$

Tend to Disagree $\quad 0$

Tend to Agree $\quad 32$

Strongly Agree 68

4. APNs should be involved in making policy decisions affecting their working conditions

Strongly Disagree $\quad 2$

Tend to Disagree $\quad 5$

Tend to Agree $\quad 48$

Strongly Agree $\quad 45$

5. APNs should be accountable to patients for the care they provide

Tend to Agree $\quad 20$

Strongly Agree $\quad 80$ 


\section{Table 3 (Continued)}

Frequency of Responses for Jefferson Scale of Attitude Questionnaire

Question item $\quad$ Percent (\%)

6. There are many overlapping areas of responsibility between physicians and APNs

Strongly Disagree $\quad 0$

$\begin{array}{ll}\text { Tend to Disagree } & 39\end{array}$

Tend to Agree $\quad 50$

Strongly Agree 11

7. APNs have special expertise in patient education and psychological counseling

Strongly Disagree $\quad 2$

Tend to Disagree $\quad 14$

Tend to Agree $\quad 59$

Strongly Agree 25

8. Doctors should be the dominant authority in all health care matters

Strongly Disagree 2

Tend to Disagree $\quad 23$

Tend to Agree $\quad 30$

Strongly Agree 45

9. Physicians and APNs should contribute to decisions regarding the hospital discharge of patients

Strongly Disagree $\quad 9$

Tend to Disagree $\quad 54$

Tend to Agree $\quad 35$

Strongly Agree $\quad 2$

10. The primary function of the APN is to carry out the physician's orders

Strongly Disagree $\quad 21$

Tend to Disagree $\quad 36$

Tend to Agree $\quad 34$

Strongly Agree 9

11. APNs should be involved in making policy decisions concerning the hospital support services upon which their work depends

Strongly Disagree $\quad 0$

Tend to Disagree $\quad 20$

Tend to Agree $\quad 59$

Strongly Agree 21

12. APNs should also have responsibility for monitoring the effects of medical treatment

Strongly Disagree $\quad 0$

Tend to Disagree $\quad 0$

Tend to Agree $\quad 43$

Strongly Agree 57

13. APNs should question a physician's order when they feel that it might have potential for detrimental effects on the patient

Strongly Disagree $\quad 0$

Tend to Disagree $\quad 0$

Tend to Agree $\quad 36$

Strongly Agree $\quad 64$ 


\section{Table 3 (Continued)}

Frequency of Responses for Jefferson Scale of Attitude Questionnaire

Question item $\quad$ Percent (\%)

14. Physicians should be educated to establish collaborative relationships with APNs

Strongly Disagree

Tend to Disagree

Tend to Agree

Strongly Agree

15. Interprofessional relationships between physicians and APNs should be included in their educational programs.

Strongly Disagree

Tend to Disagree

Tend to Agree

Strongly Agree

\section{Jefferson scale scores.}

Interpretation of the JSAPNC was based on the results of the total scale scores and the four subscale's scores. As discussed in the instrument section of methodology chapter, the total 15 question items of JSAPNC were categorized into four subscales: Shared Education/collaboration, Caring vs. Curing, APN autonomy, and physician authority. These subscales were correlated to the four underlying factors that thought to influence the attitudes toward Physician-APN collaboration. Therefore, each returned JSAPNC was analyzed based on the five scores obtained, which include a total Jefferson scale score and four subscale's scores. The descriptive statistics, with the summary of the means and standard deviations of these scores is found in Table 4.

As described, the mean of the total Jefferson Scale score for the participating physicians was $48.25(\mathrm{SD}=6.31)$. The highest possible score for a total Jefferson Scale was 60 , with the higher the score indicating the more positive attitudes toward Physician-APN collaboration. Among this group of respondents, the highest score was 59 and the lowest one was 31 . 


\section{Table 4}

Summary of Scores for the Total Jefferson Scale and Four Subscales

\begin{tabular}{lccccc}
\hline Statistical item & $\begin{array}{c}\text { Total } \\
\text { Jefferson } \\
\text { Scale Scores }\end{array}$ & $\begin{array}{c}\text { Shared } \\
\text { Education } \\
\text { Collaboration }\end{array}$ & $\begin{array}{c}\text { Caring Vs. } \\
\text { Curing }\end{array}$ & $\begin{array}{c}\text { APN } \\
\text { Autonomy }\end{array}$ & $\begin{array}{c}\text { Physician } \\
\text { Authority }\end{array}$ \\
\hline Mean & 48.25 & 20.07 & 6.43 & 17.36 & 4.50 \\
Median & 49.00 & 21.00 & 6.00 & 18.00 & 4.00 \\
Std. Deviation & 6.31 & 3.17 & 1.17 & 1.89 & 1.50 \\
Minimum & 31.00 & 11.00 & 4.00 & 14.00 & 2.00 \\
Maximum & 59.00 & 24.00 & 8.00 & 20.00 & 7.00 \\
\hline
\end{tabular}

The mean score for Shared education/Collaboration subscale was 20.01(SD=3.17, possible highest score $=24)$; for Caring vs. Curing subscale was $6.43(\mathrm{SD}=1.17$, possible highest score $=8)$; for APN Autonomy subscale was $17.36(\mathrm{SD}=1.89$, possible highest score $=20)$; and for Physician Authority subscale, after reversing the score, was $4.5(\mathrm{SD}=1.50$, possible highest score $=8)$ respectively. A higher score on the share education/ collaboration subscale shows a greater orientation toward interdisciplinary education and interprofessional collaboration. A higher score on the caring, as opposed to curing, indicates a more positive view of APNs' contributions to psychological and educational parts of patient care. A higher score on the APNs' autonomy subscale suggests more agreement with APNs' involvement in decisions on patient care and polices. A higher score on physicians' authority dimension indicates rejecting a totally dominant role of physicians in aspects of patient care.

\section{Reliability}

Prior to further investigation, a reliability analysis for the internal consistency using Cronbach's alpha was conducted for the total Jefferson Scale and its four subscales. The results are reported in Table 5. The reliability Alpha coefficients for the total modified Jefferson Scale is 0.81 . A reliability coefficient of this magnitude is considered as good for a psychometric test. 
Thus it confirmed the total modified Jefferson Scales as a measurement tool, when applied to this group of participants, is consistent and reliable. The alpha coefficients for the four subscales varied, ranging from 0.60 (Physician authority subscale) to 0.87 (for Shared education/collaboration subscale), which were within the acceptable range for attitude scales.

\section{Table 5}

Reliability Test for the Total Jefferson Scale and Four Sub-Scales

\begin{tabular}{lcr}
\hline \multicolumn{1}{c}{ Scale } & Cronbach's Alpha & $\begin{array}{c}\text { Questions Associated with } \\
\text { the Scale }\end{array}$ \\
\hline Total Jefferson Scale & .81 & $\begin{array}{r}\text { All 15 questions } \\
\text { Shared Education/Collaboration }\end{array}$ \\
Caring Vs. Curing & .87 & Question \#1,3,6,9,14,15 \\
APN Autonomy & .71 & Question \# 2, 7 \\
Physician Authority & .67 & Question \# 4, 5, 11, 12, 13 \\
\hline
\end{tabular}

\section{Analysis by Variables}

Statistical tests were performed using SPSS software to explore the potential influence of the total Jefferson scale score by the independent variables, such as gender, age, physician specialty and prior experiences with APNs. Pearson test was used to test whether age has an effect on the physician's total Jefferson score. Independent t-tests were selected to find out if there were significant differences in mean total Jefferson score based on physician's gender, primary specialty and whether they had prior working experience with APNs. Level of significance was set at 0.05 probabilities. The results of these comparison tests and $p$ values are presented on table 6 on the next page.

As reported in table 6 , the total Jefferson score was not affected by age or gender of the participants. There were no significant differences among different age groups ( $p>0.05)$, as well as male or female participants ( $p>0.05$ ). However, the mean total Jefferson score of primary care physicians $(M=50.0, S D=5.8)$ was higher than that of the specialists $(M=46.3, S D=6.4)$. And 
this difference was statistically significant with a P value equal to $0.047(p<0.05)$. In addition, the finding showed that physicians who had prior experiences working with APNs had higher total Jefferson score $(M=49.5, \mathrm{SD}=6.4)$. Again, the mean score differences was statistically significant $(\mathrm{p}=0.033)$.

Table 6

Results of Comparison Tests of Mean Total Jefferson Scores for Independent Variables Independent Variable Group $\quad$ Comparison Test $\quad$ Equality of $\quad P$ value

\begin{tabular}{lcccc} 
& & & Variance \\
\hline Age & --- & Pearson test & --- & .247 \\
Gender & Male & Independent T test & Yes & .719 \\
Specialty & Female & & & \\
& $\begin{array}{c}\text { Primary Care } \\
\text { Specialties }\end{array}$ & Independent T test & Yes & $.047^{*}$ \\
Prior experience with & Yes & Independent T test & Yes & $.033^{*}$ \\
APNs & No & & & \\
\hline
\end{tabular}

$* \mathrm{P}<.05$

Since the specialty and prior experience with APNs were two independent variables that influenced the physicians' overall attitudes toward collaboration based on the analysis of the total Jefferson score, these two independent variables were further evaluated using the four subscale scores. The investigation on the difference of the mean scores of the four subscale provided insights on which factors (correlated with the subscale) were responsible for the physicians' attitude variance.

Table 7 revealed the results of independent t-tests for the four subscales. As indicated, when comparing based on the physician's specialty, the only significant effect noted on the subscale of shared education and collaboration. When comparing based on the prior working experience with APNs, there were statistical significances showed on the subscales of Caring vs. 
Curing and APN autonomy. These results indicated that the main differences towards collaboration attitudes for primary care physicians and specialists were heavily due to their different perception toward shared education and collaboration. As for the physician groups with or without prior working experiences with APNs, the significant effects were observed mainly because of their different views on APNs' contributions to psychological and educational parts of patient care and APN autonomy.

Table 7

Results of T-tests for Sub Scales

\begin{tabular}{llcr}
\hline Group & \multicolumn{1}{c}{ Sub-Scale } & $\begin{array}{c}\text { Equality of } \\
\text { Variances }\end{array}$ & P value \\
\hline Primary Care Physicians & Shared education/Collaboration & Yes & $0.037^{*}$ \\
vs. & Caring vs. Curing & Yes & 0.067 \\
Specialists & APN Autonomy & Yes & 0.171 \\
& Physician Authority & Yes & 0.195 \\
& & & \\
\hline Prior Working Experiences & Shared education/Collaboration & Yes & 0.078 \\
with APNs & Caring vs. Curing & Yes & $0.013^{*}$ \\
Yes vs. No & APN Autonomy & Yes & $0.015^{*}$ \\
& Physician Authority & yes & 0.588 \\
\hline
\end{tabular}

$* \mathrm{P}<.05$

Finally, the relationships between physicians' attitudes toward APN collaboration and their personal/cultural beliefs on teamwork and the benefits of APN care were explored. This inspection was achieved by using Spearman's Rho correlation test to assess the correlations between variables, which were the total Jefferson score (scale variable) and the Liker-scale responses to the six researcher-generated questions (ordinal variable). Each one of the six researcher-generated questions (ordinal variable) was measured to evaluate their strength and direction of association with the total Jefferson score. Table 8 summarized the Spearman correlation coefficient and the $\mathrm{P}$ values for the variables accordingly. 


\section{Table 8}

Results of Spearman's Rho Correlation test for Researcher-generated questions

\begin{tabular}{lcc}
\hline \multicolumn{1}{c}{ Variable } & Correlation Coefficient & P value \\
\hline I work well with my colleagues and support staff & 0.202 & 0.187 \\
My culture promotes harmony and team work & 0.116 & 0.454 \\
My office/organization supports utilization of APNs & 0.493 & $0.001^{*}$ \\
I believe my patients are acceptable to the APN's care & 0.564 & $0.000^{*}$ \\
I believe my patients can benefit from APNs' care & 0.483 & $0.001^{*}$ \\
I plan to hire or work with APNs if my practice expands & 0.566 & $0.000^{*}$ \\
\hline$* \mathrm{P}<.05$ & &
\end{tabular}

The results of the Spearman's Rho Correlation tests indicated there were no significant correlation between the total Jefferson score and the physician's personal or cultural beliefs on teamwork and harmony working environment. There were, however, moderate levels of positive correlations between the total Jefferson scores and how supportive the organization was towards the utilization of APNs, as well as how much the physician believed in the benefits of APNs' care. The more supportive the physician's office/organization was toward APN utilization, the higher the physician's total Jefferson score. If the physician believed his/her patients were acceptable and could benefit from the APNs' care, he/she tended to rank higher on the total Jefferson score. In addition, strong correlation was found on the total Jefferson score and the physician's decision to hire APNs. Physician who planned to hire APNs was more than likely to score higher on the total Jefferson score and vice versa.

\section{Discussion}

The descriptive findings of this study showed that the CCHCA physicians $(N=44)$ have a moderate positive attitude toward Physician-APN collaboration with a mean total Jefferson score of $48.3(\mathrm{SD}=6.3)$. This is comparable to Hojat Et al. (2003) findings in a sample of American physicians $(\mathrm{N}=118)$ where he showed that the average mean score toward physician-nurse collaboration was $48(\mathrm{SD}=4.9)$. Although the study of Hojat et al. (2003) did not specify the ethnicity of the participants, it is reasonable to assume that the majority of them were Caucasian 
physicians based on the location of the study site. When comparing to this study's respondents where $90 \%$ were Chinese, it is rational to say that this group of Chinese-American physicians' perception toward collaboration is similar to what have reported in the literature before. However, it is hard to conclude that ethnicity is not a factor that impacts a physician's attitude towards collaboration with APNs. A better study design is needed in the future to generate more reliable data.

Gender or age seems to make no difference on CCHCA physicians' view towards Physician-APN collaboration. There were no positive or negative correlations found between gender and age with the total Jefferson score. It indicates that these two demographic variables most likely are not prevailing factors in forming attitudes toward Physician-APN collaboration. Similar conclusions were noted in several similar studies in the past (Hojat et al., 2003; Jones \& Fitzpatrick, 2009).

CCHCA physicians who work in primary care settings, including family practice, internal medicine, OBGYN, and pediatric medicine, tend to have more positive attitudes toward Physician-APN collaboration than their colleagues who work in the subspecialty field. Of the four subscales that contribute to the total Jefferson scale, primary care physicians scored significantly higher on the subscale of "shared education and collaboration" compare to the specialists. This finding suggests that primary care physicians generally are more certain of the value of shared education and interprofessional collaboration than the specialists. Possible explanation for this pattern includes:

- some primary care physician have already working with the APNs so they see the needs and the benefits of shared education and collaboration; 
- many primary care physicians will need to collaborate with APNs more often in order to handle the increased service demand in their practice due to the activation of Obama Care, therefore, they have an urge to promote shared education and collaboration;

- APNs are less utilized and desired in the specialty practice which results in the specialists view the concepts of shared education and collaboration less important. In conclusion, more work is needed to educate the physicians working in the specialty filed in order to endorse shared education and collaboration in all professions. Again, further empirical examination of this finding is necessary to determine and confirm its significance.

Lack of significant correlation between individual/cultural beliefs on harmony and teamwork with the total Jefferson score suggested that personal and cultural influences have no strong effect on CCHCA physicians' attitude toward physician-APN collaboration. On the other hand, physicians with prior working experiences with APNs expressed higher positive attitudes towards Physician-APN collaboration. Specifically, they scored higher on the factors of "Caring vs. Curing" and APN autonomy. Hence, prior exposure to working with APNs provided the physicians an opportunity to better understand the educational and psychological expertise of APNs and support APN autonomy. In addition, the study revealed that physicians whose office or health organization was supportive in utilization of APNs were likely to have more positive perceptions regarding collaboration. Likewise, physicians who believed their patients were acceptable and could benefit from APNs' care exhibited more optimistic attitudes on collaborative practice with APNs. Consequently, these physicians were more likely to hire or work with APNs if their practice expands. Overall, these findings indicate that personal and interpersonal influences are relatively weak factors, whereas the professional and organizational influences have more substantial effect on changing the collaboration attitude. 
It is worth mentioning that the majority of CCHCA physicians strongly believed in physician authority. This was evidenced by the fact that nearly half of the physicians (43\%) believed the primary function of the APN is to carry out the physicians' orders and three quarter of the physicians (75\%) agreed that doctors should be the dominant authority in all health care matters. This finding suggests that the vision of "physician leading power" is still deeply rooted in this group of physicians. Although the result is disenchanting, it is not surprising as traditional Chinese culture commonly endorses a hierarchical model of professional roles, in which the nursing profession is subordinate to the medicine profession. And nurses have little autonomy whereas physicians have total governance in patient care decisions. Further study in Chinese or other Asian community is needed to better understand this pattern so that problems can be addressed to encourage a complementary professional role model in the U.S. healthcare society.

However, it is interesting to know that most of physicians agreed that doctors should be educated to establish collaborative relationship with APNs and such education should be included in their educational programs. This finding showed that physicians are indeed aware of the deficiency in their knowledge about interproessional collaboration and they see the needs and the benefit of such education occur early in their educational training program. Providing interporfessional education training courses thus is necessary and essential in order to promote effective collaborative practice between physicians and APNs.

\section{Limitations}

The convenience sampling used in this study may limit the generalization of the findings to a broader population of physicians. A descriptive, self-reported survey design and the study's low response rate (20\%) also affected its generalizability of the findings. Lack of study incentives could have decreased the study's response rate and increased bias since the 
respondents were more likely to have stronger opinions and special interests in this topic. Although the internal reliability of the total modified Jefferson Scales and four subscales were found to be acceptable, the alpha coefficients of the two subscales belonged to the lower range of normal limit. Thus, the internal consistency aspect of reliability of subscales "APN autonomy" and "Physician Authority" were relatively weak. Hence, more validity evidence is needed. The internal validity of the findings may be improved by having a larger sample size in the future studies. In addition, more data on score stability over time is needed, for instance, collecting JSAPNC scores initially, and at 3,6 months interval after education sessions to check test-retest reliability. Future replication of the study in a similar cultural community can also provide assurances for the external validity (generalizability) of the findings.

\section{Future Implications for Practice}

The influence of ethnicity, as a factor, on the physicians' attitudes toward collaboration with APNs was inconclusive in this study. However, the findings provided some needed information on Chinese-American physicians' viewpoints toward Physician-APN collaboration. It is undeniable that culture background and stigma somehow affect ones' behaviors and perception-- healthcare providers like physicians are not excluded. More evidences and research are required to better comprehend the cultural impact on both physicians and APNs toward interprofessional collaboration.

This project provides some insight to the attitude of Chinese physicians toward collaboration with APNs in a Chinese community of an urban city. It also served as a needs assessment for the CCHCA and the findings confirm that the collaboration knowledge deficiency exists and the interprofessional collaboration training is warranted. Focus groups or individual interviews may be carried out in the near future to explore the barriers to the collaborative 
relationship within the organization and hoping to create valuable strategies to improve interprofessional collaboration. Consequently, online or in-person educational sessions can be conducted in CCHCA as the extended step of this project.

Moreover, the study findings suggest that an amiable personality or a cultural preference on harmony relationship and teamwork do not automatically lead to a better understanding and desire for interprofessional collaboration. Professional and organizational influences are more powerful means to affect one's attitude toward collaboration. The study findings also alleged that a hierarchical structure existed in the health professions within the Chinese community, which is a major drawback element to the positive attitude toward Physician-APNs collaboration. This again supports conclusions from previous studies stating that physician domination was a strong barrier that detriments Physician-APNs collaboration. Shared education and common working experiences can provide APNs the opportunity to modify socially prescribed stereotypical roles and foster mutually respectful interprofessional relationships between nursing and medical professionals. Hence, APNs should actively engage in interprofessional education to endorse positive changes in interprofessional collaboration in their clinical practices.

Indeed, interprofessional collaboration in any culture benefits patients and contribute to better communication and satisfaction within the professions (Rosenstein, 2002). Since there are many overlapping areas of practices and responsibilities that exist between physicians and APNs, such collaborative relationship is even more imperative for quality of care and team effectiveness of both professions. APNs have a long way to go before reaching shared responsibility, equal authority and true autonomy in the health care system. It is essential for us to advocate for the rights of our nursing profession and promote a complementary model of professional roles in any 
community within our society. APNs should also continue to support research and activities that encouraging interprofessional collaboration and seek for innovative approaches to improve interprofessional collaboration in our practices. 


\section{References}

Almost, J., \& Laschinger, H. (2002). Workplace empowerment, collaborative work relationships, and job strain in nurse practitioners. Journal of the American Academy of Nurse Practitioners, 14, 408-420.

Bailey, P., Jones, L., \& Way, D. (2005). Family physician/nurse practitioner: Stories of collaboration. Journal of Advanced Nursing, 53, 381-391.

Baruch, Y., \& Holtom, B. (2008). Survey response rate levels and trends in organizational research. Human Relations, 61(8), 1139-1160.

Beal, J., \& Freda, M. (2004). Attitudes and beliefs for effective pediatric nurse practitioner and physician collaboration. MCN, the American Journal of Maternal/Child Nursing, 29(5), 332.

Börkan, B. (2010). The mode effect in mixed-mode surveys: Mail and web surveys. Social Science Computer Review, 28(3), 371-380.

Brennan, M., \& Charbonneau, J. (2009). Improving mail survey response rates using chocolate and replacement questionnaires. The Public Opinion Quarterly, 73(2), 368-378.

Buppert, C. (2007). Implications for collaborating physicians. Journal of Nurse Practitioners, 3, 306-308.

Clarin, O. (2007). Strategies to overcome barriers to effective nurse practitioner and physician collaboration. The Journal for Nurse Practitioners, 3 (8), 538-548. 
Coeling, H. V., \& Cukr, P. L. (2000). Communication styles that promote perceptions of collaboration, quality, and nurse satisfaction. Journal of Nursing Care Quality, 14, 6374.

Corser, W. D. (1998). A conceptual model of collaborative nurse-physician interactions: The management of traditional influences and personal tendencies. Scholarly Inquiry for Nursing Practice, 12, 325 - 341 .

Cowan, M. J., Shapiro,M., Hays, R. D., Afifi, A., Vazirani, S., \& Ward, C. R. (2006). The effect of a multidisciplinary hospitalist/physician and advanced practice nurse collaboration on hospital costs. Journal of Nursing Administration, 36, 79-85.

Dougherty, M., \& Larson, E. (2005). A review of instruments measuring nurse-physician collaboration. JONA: The Journal of Nursing Administration, 35(5), 244-253.

Fletcher, C., Baker, S., Copeland, L., Reeves, P., \& Lowery, J. (2007). Nurse practitioners' and physicians' views of nps as providers of primary care to veterans. Journal of Nursing Scholarship : An Official Publication of Sigma Theta Tau International Honor Society of Nursing / Sigma Theta Tau, 39(4), 358-362.

Gilbert, J., Yan, J., \& Hoffman, S. (2010). A who report: Framework for action on interprofessional education and collaborative practice. Journal of Allied Health, 39(3 part 2), 196.

Gardner, D. R. (2005). Ten lessons on collaboration. Online Journal of Issues in Nursing, 10, 1 17. 
Haskins, A. (2008). An Exploration of Satisfaction, Psychological Stress, and Readiness for Interprofessional Learning in Medical, Nursing, Allied Health, and Social Work Students in an Interprofessional Health Care Course. n.p.: ProQuest, UMI Dissertations Publishing.

Hader, R. (2005). Collaboration paces the way for better care. Nurse Management, 36, 4.

Hendel, T., Fish, M., \& Berger, O. (2007). Nurse/physician conflict management mode choices: Implications for improved collaborative practice. Nursing Administration Quarterly, 31, 233-253.

Hojat, M., Herman, M. (1985). Developing an instrument to measure attitudes toward nurses: Preliminary psychometric findings. Psychological Reports, 56, 571-579.

Hojat, M., Fields, S., Veloski, J., Griffiths, M., Cohen, M., \& Plumb, J. (1999). Psychometrics properties of an attitude scale measuring physician-nurse collaboration. Evaluation \& Health Professions, 22 (2), 208-220.

Hojat, M., Gonnella, J., Nasca, T., Fields, S., Cicchetti, A., et al. (2003). Comparisons of american, israeli, italian and mexican physicians and nurses on the total and factor scores of the jefferson scale of attitudes toward physician-nurse collaborative relationships. International Journal of Nursing Studies, 40(4), 427-435.

Hojat, M., Nasca, T., Cohen, M., Fields, S., Rattner, S., et al. (2001). Attitudes toward physiciannurse collaboration: A cross-cultural study of male and female physicians and nurses in the united states and mexico. Nursing Research, 50(2), 123-128. 
Homans, G. (1958). Social behavior as exchange. American Journal of Sociology, 63(6), 597606.

Jones, T., \& Fitzpatrick, J. (2009). Crna-physician collaboration in anesthesia. AANA Journal, 77(6), 431 .

Kaplowitz, M. , Hadlock, T. , \& Levine, R. (2004). A comparison of web and mail survey response rates. The Public Opinion Quarterly, 68(1), 94-101.

Leiter, M. P., \& Laschinger, H. K. S. (2006). Relationships of work and practice environment to professional burnout. Nursing Research, 55, 137-146.

Leonard, M., Graham, S., \& Bonacum, D. (2004). The human factor: The critical importance of effective teamwork and communication in providing safe care. Quality and Safety in Healthcare, 13(Suppl. 1), 85-90.

Lindeke, L. L., \& Siekert, A. M. (2005). Nurse-physician workplace collaboration. Online Journal of Issues in Nursing, 10, 1-15.

Lopez, K. (2008). A Mixed Methods Study of Nurse-physician Work Relationships. n.p.: ProQuest, UMI Dissertations Publishing.

MacDonald, J. (2001). Physicians' Perceptions of Nurse Practitioners and Collaboration. n.p.: ProQuest, UMI Dissertations Publishing.

Moore, J., \& Prentice, D. (2013). Collaboration among nurse practitioners and registered nurses in outpatient oncology settings in canada. Journal of Advanced Nursing, 69(7), 15741583. 
Moore-Smithson, J. (2005). The Association Between Nurse-physician Collaboration and Satisfaction with the Decision-making Process in the Ambulatory Care Setting. n.p.: ProQuest, UMI Dissertations Publishing.

Oberle, K., \& Tenove, S. (2000). Ethical issues in public health nursing. Nursing Ethics, 7(5), $425-438$

O'Brien, J., Martin, D., Heyworth, J., \& Meyer, N. (2009). A phenomenological perspective on advanced practice nurse-physician collaboration within an interdisciplinary healthcare team. Journal of the American Academy of Nurse Practitioners, 21(8), 444-453.

Phillips, R., Green, L., Fryer, G., \& Dovey, S. (2001). Trumping professional roles; Collaboration of nurse practitioners and physicians for a better U.S. health care system. American Family Physician, 64, 1325.

Rosenstein, A. H. (2002). Nurse-physician relationships: Impact on nurse satisfaction and retention. American Journal of Nursing, 102, 26-34.

Rosenstein, A. H., \& O'Daniel, M. (2005). Disruptive behavior and clinical outcomes: Perceptions of nurses and physicians. American Journal of Nursing, 105, 54-64.

Schadewaldt, V., McInnes, E., Hiller, J., \& Gardner, A. (2013). Views and experiences of nurse practitioners and medical practitioners with collaborative practice in primary health care an integrative review. BMC Family Practice, 14(1), 132.

Sievers, B., \& Wolf, S. (2006). Achieving clinical nurse specialist competencies and outcomes through interdisciplinary education. Clinical Nurse Specialist, 20, 75-80.

Sirota, T. (2007). Nurse-physician relationships: Improving or not? Nursing, 2007 37, 52-55. 
Shih, T., \& Fan, X. (2008). Comparing response rates from web and mail surveys: A metaanalysis. Field Methods, 20(3), 249-271.

Stein-Parbury, J., \& Liaschenko, J. (2007). Understanding collaboration between nurses and physicians as knowledge at work. American Journal of Critical Care, 16, 470-478.

Street, D., \& Cossman, J. (2010). Does familiarity breed respect? physician attitudes toward nurse practitioners in a medically underserved state. Journal of the American Academy of Nurse Practitioners, 22(8), 431-439.

Wright, K. (1997). Advanced practice nursing: Merging the clinical nurse specialist and nurse practitioner roles. Gastroenterology Nursing, 20, 57-60.

Zander, P. (2005). Attitudes of Physicians and Advanced Practice Nurses Toward Collaborative Practice. n.p.: ProQuest, UMI Dissertations Publishing. 


\section{Appendix A}

\section{CCHCA Permission letter for the project}

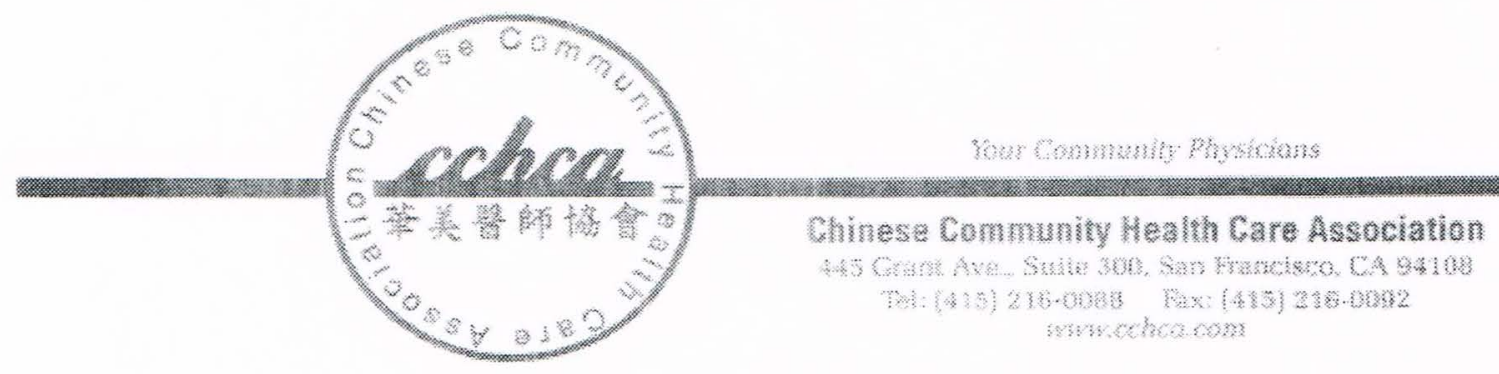

Research with Hurran Subiects Protocol Review

wo:

Ms. Wy Tan

940 stoney court

Mubrae, Cs 94030

From: Polly Chen, Difector of Operations

Date: April 24, 2014

Protocol Tite: Physicians' perception toward collaborative pactice with Nurse Practitioners

Dear Ms. Tans

I have zound you proposal to be savistactory. This latter shall be considered notification to you of our decision and that you may proceed with the data collection. Physician participation in your research study survey is voluntary and mainy up to the individual physician's consent. The CCHCA can provide you in suppont of your dissertation research:

- To promote your research study on our newsletter

- To assist vou with sending out the research sunvey questionaire to the physicians in the CCHCA

CCHCA whll only assist with the tasks listed above and whl not be responsible for any research functions beyond these responsiblities. CCHCA whil not be held responsible for any collection of actral research data, nor be responsible for any administrative requirements of this wroject.

Please contact me with any further questions you may have. 1 look forward to working with you on this research project.

Sincerely

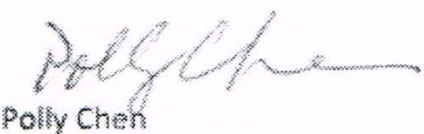

poly chen

Director of Operations

chinese Community Health Care Association 


\section{Appendix B}

Permission Letter to use Jefferson Scale of Attitude

Mohammadreza Hojat «Mohammadraza. Holat@jefferson.edu

\section{Dear Ivy:}

In response to your request, attached I am sending you a copy of the scale, its scoring instructions, and a few relevant articles.

You have my permission to use the scale in your not-for-profit research, given that proper credit will be given to the original source(s) and the Jefferson copyright sign will be printed on any copy of the scale you will be using in your project.

I wish you good luck with your project, and please inform me of your progress.

( - :

Hojat

- Mohammadreza Hojat, wh.2.

- Research Protessor of Psychiatry and Human Behavior

- Director of Jetferson Longitudinal Study

- Center for Research in Medical Education and Health Care

- Jefferson Medical College, Curtis Building

- 1015 Walnut Street, $3^{\text {rs }}$ Foor, Suite 320

- Phindelpha, para 1907, USA

\%

- Voice-mail: (215) 955-9459

- Fax: (215) 923-6939

- Emall Mohammadreza.Molat@jefterson.edu

- Website: www. Ŝlu edu/mo/crmehc

Webpage: http://www.jefferson.edu/imc/crmehc/faculty/faculty/hojat.html

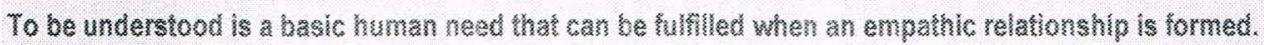

Information about the book "Empathy in patient Care" and experts' comments are posted at:

www soringer.com 10 387 . 33607.9 (publisher)

CONFDENTALTY NOTE: The documents) accompanying this transmission moy contain confidentiol information. This information is htended for the use of the individubls nomed above. If you hove received this information in errot, please notify me immediately and arranged for the retum or destuction of the dacuments). 


\author{
Appendix C \\ California State University, \\ Fresno Department of Nursing \\ IRB Approval letter
}

Date: June 20, 2014

\title{
RE: DNP-1400 Chinese Community Health Care Association Physicians' perception on Physician-APN Collaboration.
}

Dear Ivy Tan,

As the Chair of the Department of Nursing Research Committee, serving as the Institutional Review Board for the Department of Nursing, I have reviewed and approved your review request for the abovereferenced project for a period of 12 months. I have determined your study to meet the criteria for Minimal Risk IRB review.

Under the Policy and Procedures for Research with Human Subjects at California State University, Fresno, your proposal meets minimal risk criteria according to section 3.3.7: Research in which the risks of harm anticipated are not greater, probability and magnitude, than those ordinarily encountered in daily life or during the performance of routine physical or psychological examinations or tests.

The Research Committee may periodically wish to assess the adequacy of research process. If, in the course of the study, you consider making any changes in the protocol or consent form, you must forward this information to the Research Committee prior to implementation unless the change is necessary to eliminate an apparent immediate hazard to the research participant(s).

This study expires: June 20, 2015

The Research Committee is authorized to periodically assess the adequacy of the consent and research process. All problems having to do with subject safety must be reported to the Research Committee. Please maintain proper data control and confidentiality.

If you have any questions, please contact me through the CSU, Fresno Department of Nursing Research Committee at tereag@csufresno.edu.

Sincerely,

Terea Giannetta, DNP

Department of Nursing, Research Committee, Chair 


\section{Appendix D}

\section{Initial Recruitment letter to physicians}

June 1, 2014

Dear Physician:

We are conducting a study assessing Chinese physicians' attitudes towards collaboration with advanced practice nurses (APNs), which include nurse practitioners, nurse midwives, clinical nurse specialists, and nurse anesthetists. To better understand this topic we would like your opinion.

Enclosed is a two-paged survey that may take about 5 to 10 minutes to be finished. Your responses will be kept entirely confidential and you will not be personally identified. Your participation in this study is entirely voluntary and there are no adverse consequences should you choose to not participate.

Your participation in this survey is important to us. The findings will attempt to fill the knowledge gap on this topic and provide insight about the attitude of physicians toward collaboration with APNs in the Chinese community. It could impact future education with regard to fostering interprofessional collaborative relationships in the community. And ultimately, the knowledge generated from this study can be utilized to promote inter-professional collaborative practice in the Chinese community in order to ensure safe, high quality patient care, and better patient outcomes.

If you agree to participate in this study please complete the survey and return it in the self-addressed stamped envelope provided. Completion of the survey will imply consent for this project.

Should you have any questions regarding the study and would like to be informed about the final findings of the study in the future, please feel free to contact us.

Sincerely,

\section{Danette Dutra}

Danette Dutra, EdD, FNP-C

Assistant Professor, School of Nursing

California State University, Fresno

Primary Investigator

ddutra@csufresno.edu

559-278-5615

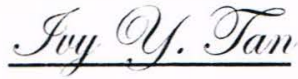

Ivy Tan, DNP(c), FNP-C

Doctoral Student

California State University, Fresno

Co-Investigator

ivyingt@mail.csufresno.edu

415-370-5376 


\section{Appendix E \\ Follow-up Recruitment letter to physicians}

August 14, 2014

Dear Physician:

We are following up on a recent mail survey sent to you assessing CCHCA physicians' attitudes towards collaboration with advanced practice nurses (APNs). If you have already mailed back your response, we thank you for your participation and you do not need to repeat the survey online again this time.

If you have not responded to the previous mail survey, we hope you can consider taking about 5 to 10 minutes to be complete this survey online by press Control + Click to follow the link here:

https://www.surveymonkey.com/s.aspx?sm=tpS/sQCi8MPRwBedUfkcZw 3d 3d.

Completion of the survey will imply your consent for this study.

Your responses will be kept entirely confidential and you will not be personally identified. Your participation in this study is entirely voluntary and there are no adverse consequences should you choose to not participate.

Your participation in this survey is very important to us. The findings will attempt to fill the knowledge gap on this topic and provide insight about the attitude of physicians toward collaboration with APNs in the Chinese community. It could impact future education with regard to fostering inter-professional collaborative relationships in the community. And ultimately, the knowledge generated from this study can be utilized to promote inter-professional collaborative practice in the Chinese community in order to ensure safe, high quality patient care, and better patient outcomes.

Should you have any questions regarding the study and would like to be informed about the final findings of the study in the future, please feel free to contact us.

Sincerely,

Danette Dutra

Danette Dutra, EdD, FNP-C

Assistant Professor, School of Nursing

California State University, Fresno

Primary Investigator

ddutra@csufresno.edu

559-278-5615
You, QY. San

Ivy Tan, DNP(c), FNP-C

Doctoral Student

California State University, Fresno

Co-Investigator

ivyingt@mail.csufresno.edu

$415-370-5376$ 


\section{Appendix F}

Researcher-generated survey questionnaire

\section{Chinese Community Health Care Association Physicians' perception on physician-advanced practice nurse (APN) collaboration Survey Questionnaire}

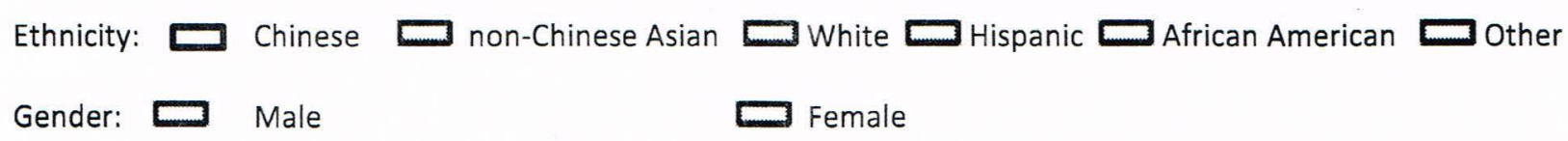

Age (in years):

Primary Specialty:

Have you worked or been working with APNs in the clinics or hospitals: $\square$ Yes $\square$ No

Strongly

Agree Agree
Strongly

Disagree

Disagree

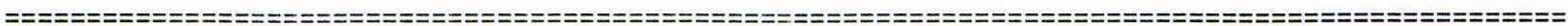

1. I work well with my colleagues

and support staff.

2. My culture promotes harmony and team work.

3. My office/organization supports utilization of mid-level practitioners, such as APNs.

4. I believe my patients are acceptable to the APN's care.

5. I believe my patients can benefit from APNs' care.

6. I plan to hire or work with APNs if my practice expands.
4

4

4

4

4

4
3

1
5

5

5

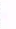

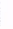

3

2

1

(1)

3

2

1 


\section{Appendix G}

\section{JEFFERSON SCALE OF ATTITUDES TOWARD PHYSICIAN-APN COLLABORATION}

INSTRUCTIONS: Please indicate the extent of your agreement or disagreement with each of the following statements by circling the appropriate number. For the purposes of this survey, APN is defined as "advanced practice nurse" who is prepared with advanced didactic and clinical education, knowledge, skills, and scope of practice in nursing. APN education forms the basis of four recognized general areas of specialization: Nurse practitioners (NPS), Nurse Midwives (in the United States, Certified Nurse Midwives or CNMs), Clinical nurse specialists (CNSs) and Nurse anesthetists (in the United States, Certified Registered Nurse Anesthetists or CRNAs)

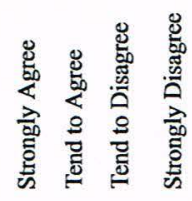

1 An APN should be viewed as a collaborator and colleague with a physician rather than his/her assistant $\quad 4 \quad 3 \quad 3 \quad 2 \quad 1$

2 APNs are qualified to assess and respond to psychological aspects of patients' needs. $\begin{array}{llll}4 & 3 & 2 & 1\end{array}$

3 During their education, medical and nursing students should be involved in teamwork in order to understand their respective roles. $\begin{array}{llll}4 & 3 & 2 & 1\end{array}$

4 APNs should be involved in making policy decisions affecting their working conditions. $\begin{array}{llll}4 & 3 & 2 & 1\end{array}$

$\leq$ APNs should be accountable to patients for the care they provide. $\begin{array}{llll}4 & 3 & 2 & 1\end{array}$

$\epsilon$ There are many overlapping areas of responsibility between physicians and APNs $\begin{array}{llll}4 & 3 & 2 & 1\end{array}$

7 APNs have special expertise in patient education and psychological counseling. $\begin{array}{llll}4 & 3 & 2 & 1\end{array}$

$\varepsilon$ Doctors should be the dominant authority in all health care matters. $\begin{array}{llll}4 & 3 & 2 & 1\end{array}$

c. Physicians and APNs should contribute to decisions regarding the hospital discharge of patients......

$\begin{array}{llll}4 & 3 & 2 & 1\end{array}$

1C The primary function of the APN is to carry out the physician's orders. $\begin{array}{llll}4 & 3 & 2 & 1\end{array}$

11 APNs should be involved in making policy decisions concerning the hospital support services upon which their work depends. $\begin{array}{llll}4 & 3 & 2 & 1\end{array}$

12 APNs should also have responsibility for monitoring the effects of medical treatment. $\begin{array}{llll}4 & 3 & 2 & 1\end{array}$

13 APNs should question a physician's order when they feel that it might have the potential for detrimental effects on the patient.

14 Physicians should be educated to establish collaborative relationships with APNs.

15 Interprofessional relationships between physicians and APNs should be included in their educational programs.

(C) Jefferson Medical College, 2001. All rights reserved. Reprinted by permission from Mohammadreza Hojat, Ph.D. 